\title{
A theoretical framework for the site-specific and frequency-dependent neuronal effects of deep brain stimulation
}

\author{
Luka Milosevic ${ }^{1,2,3,4, *}$, Suneil K Kalia ${ }^{1,3,4,5,6}$, Mojgan Hodaie ${ }^{1,4,5,6}$, Andres M Lozano ${ }^{1,4,5,6}$, Milos R \\ Popovic $^{2,3,4}$, William D Hutchison ${ }^{4,6,7 *}$, Milad Lankarany ${ }^{1,2,3, *}$ \\ ${ }^{1}$ Krembil Research Institute, University Health Network, Toronto, Canada \\ ${ }^{2}$ Institute of Biomedical Engineering, University of Toronto, Toronto, Canada. \\ ${ }^{3}$ KITE, Toronto Rehabilitation Institute, University Health Network, Toronto, Canada. \\ ${ }^{4}$ CRANIA, University Health Network and University of Toronto, Toronto, Canada. \\ ${ }^{5}$ Division of Neurosurgery, Toronto Western Hospital, University Health Network, Toronto, Canada. \\ ${ }^{6}$ Department of Surgery, University of Toronto, Toronto, Canada. \\ ${ }^{7}$ Department of Physiology, University of Toronto, Toronto, Canada. \\ * indicates equal contributions
}

\section{Correspondence:}

luka.milosevic@mail.utoronto.ca (@lukamneuro)

milad.lankarany@uhnresearch.ca (@mlankarany) 


\begin{abstract}
With the growing interest in the expansion of deep brain stimulation indications, we aimed to provide experimental and computational insights into the brain-region-specific and frequency-dependent effects of extracellular stimulation on human neuronal activity. Experimentally, we demonstrated microstimulation-evoked excitatory neuronal responses in the ventral intermediate nucleus and reticular thalamus, and inhibitory responses in the subthalamic nucleus and substantia nigra pars reticulata; hypothesized to be the result of simultaneous activations of convergent afferent inputs. Higher stimulation frequencies led to a loss of site-specificity and convergence towards neuronal suppression; hypothesized to be mediated by synaptic depression. These experimental findings were reproduced by a computational framework in which relative distributions of convergent excitatory/inhibitory afferents were embedded within a model of short-term synaptic plasticity for the prediction of site-specific and frequency-dependent responses to extracellular stimulation. This theoretical framework may aid in the design of physiologically-informed stimulation paradigms in existing or prospective deep brain stimulation indications.
\end{abstract}

\title{
Tables of contents
}

$\begin{array}{ll}\text { Introduction } & 3\end{array}$

$\begin{array}{lc}\text { Results } & 5\end{array}$

$\begin{array}{lr}\text { Discussion } & 9\end{array}$

Fig. 1 - Experimental (A) peristimulus responses and (B) frequency response functions 16

Fig. 2 - Experimental time-domain responses to stimulation trains 17

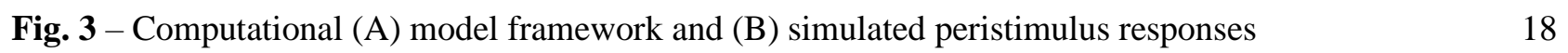

Fig. 4 - Computational time-domain synaptic currents 19

Fig. 5 - Computational time-domain membrane potential for Vim 20

Fig. 6 - Computational time-domain membrane potential for STN 21

Fig. 7 - Computational time-domain membrane potential for $\mathrm{SNr} \quad 22$

$\begin{array}{ll}\text { Methods } & 23\end{array}$

$\begin{array}{ll}\text { Acknowledgements } & 28\end{array}$

$\begin{array}{ll}\text { Author contributions } & 28\end{array}$

$\begin{array}{ll}\text { Declaration of interests } & 28\end{array}$

$\begin{array}{ll}\text { Funding } & 28\end{array}$

$\begin{array}{ll}\text { Supplementary material information } & 29\end{array}$

$\begin{array}{ll}\text { References } & 30\end{array}$ 


\section{Introduction}

Deep brain stimulation (DBS) is an established neuromodulatory therapy for several movement disorders including Parkinson's disease (Limousin et al., 1998), essential tremor (Dallapiazza et al., 2019), and dystonia (Hung et al., 2007). DBS has also recently received approvals for the treatment of obsessivecompulsive disorder (Menchón et al., 2019) and epilepsy (Fisher et al., 2010). Despite a rapidly growing interest in the development of new DBS indications (Youngerman et al., 2016), the various ways in which DBS may influence neuronal activity are not fully understood. In addition to appropriate patient selection, physiologically-informed target selection and subsequent stimulation programming play important roles in the development of novel indications (Brocker et al., 2017). As such, it must be considered that the effects of electrical stimulation in one area of the brain may differ from the effects of stimulation delivery in another (Basu et al., 2019). The objective of this study was to demonstrate how the neuronal effects of DBS vary depending on the stimulation target region and the frequency of stimulation pulses being delivered. Knowledge of the site-specific abilities to selectively upregulate or downregulate neuronal output is of importance for the development of physiologically-informed stimulation paradigms in existing or prospective DBS indications and could also aid in otherwise empirically-performed stimulation programming procedures.

It was previously demonstrated that single pulses of electrical stimulation delivered to the substantia nigra pars reticulata $(\mathrm{SNr})$ or globus pallidus internus (GPi) were associated with stimulation-evoked inhibitory responses, likely mediated by local GABA release (Dostrovsky et al., 2000; Liu et al., 2012; Milosevic et al., 2018a); whereas high-frequency stimulation (HFS) of the thalamic ventral intermediate nucleus (Vim) elicited brief short-latency excitatory responses, likely the result of unsustained local glutamate release (Milosevic et al., 2018b). In the current study, microelectrode recordings of single-neuron activity across four brain regions (Vim, thalamic reticular nucleus (Rt), subthalamic nucleus (STN), and SNr) were assessed during microstimulation trains across a range of frequencies $(1-100 \mathrm{~Hz})$. We hypothesized that (i) the effects of individual electrical stimulation pulses would vary with respect to the distribution of afferent inputs converging on target neurons (whether predominantly inhibitory or excitatory), and that based on these local neuroanatomical properties, stimulation pulses would elicit either net inhibitory or excitatory responses. Moreover, based on previous findings of HFS-induced depression of stimulusevoked field potentials (Liu et al., 2012; Milosevic et al., 2018a; Steiner et al., 2019), we hypothesized that (ii) suppression of neuronal activity during HFS is mediated by changes to short-term synaptic 
dynamics; namely, non-selective synaptic depression (i.e. depression of both inhibitory and excitatory synaptic transmission). Indeed, experimental work in rodent STN slices has demonstrated that pharmacologically-isolated excitatory and inhibitory postsynaptic potentials were both depressed during HFS (Steiner et al., 2019).

In addition to experimental data collection, we developed a computational framework for the prediction of site-specific and frequency-dependent neuronal responses to electrical stimulation based on the above hypotheses. Previous theoretical works suggest that individual pulses of extracellular stimulation (i.e. DBS) initiate action potentials which are propagated along the axons of stimulated neurons (Anderson et al., 2018); this includes both efferent axons and afferent axons and/or their terminals (Jakobs et al., 2019; McIntyre et al., 2004). These axonal activations can in turn mediate synaptic transmission. To this end, it was shown that HFS may reduce synaptic transmission fidelity by way of synaptic depression (Farokhniaee and McIntyre, 2019) or axonal failure (Rosenbaum et al., 2014). What has not typically been considered in previous computational work is that anatomical differences across stimulation sites may incur dissimilar postsynaptic responses (i.e. neuronal excitation vs. inhibition). Based on our first hypothesis, our computational model considers that the postsynaptic neuronal responses to individual DBS pulses are the result of a simultaneous activation of presynaptic inputs and takes into consideration the site-specific proportions of inhibitory and excitatory inputs converging on target neurons (derived from anatomical/ morphological literature). In accordance with our second hypothesis, the Tsodyks-Markram (TM) model (Tsodyks and Markram, 1997) of short-term synaptic plasticity was embedded within our computational framework in order to account for changes to synaptic transmission fidelity based on the frequency of successive stimuli. Despite the relative success of previous models in explaining the mechanisms of high-frequency DBS amongst homogenous cell-types, to the best of our knowledge, no theory has addressed the biophysical mechanisms underlying DBS at different frequencies across various brain regions within a single computational framework; especially within the context of the in vivo human brain. 


\section{Results}

Experimental: Responses to single stimulation pulses

The responses to single stimulation pulses (Fig. 1A) revealed stimulus-evoked excitatory responses for Vim and Rt, and inhibitory responses for STN and SNr. For Vim, the average firing rates of the immediate $20 \mathrm{~ms}(\mathrm{p}=.002)$ and 40ms ( $\mathrm{p}=.003)$ periods following stimulation pulses were significantly greater than the $20 \mathrm{~ms}$ pre-stimulus period. This was also the case for the $20 \mathrm{~ms}(\mathrm{p}<.001)$ and $40 \mathrm{~ms}(\mathrm{p}<.001)$ post-stimulus periods for Rt. For STN, the average firing rates of the $20 \mathrm{~ms}(\mathrm{p}<.0001)$ and $40 \mathrm{~ms}(\mathrm{p}=.003)$ post-stimulus periods were significantly less than the $20 \mathrm{~ms}$ pre-stimulus period. This was also the case for the $20 \mathrm{~ms}$ $(\mathrm{p}<.0001)$ and 40ms ( $<<.001)$ post-stimulus periods for SNr. All statistics have been corrected for multiple comparisons. Cohen's d $\mathrm{z}_{\mathrm{z}}$ effect sizes are depicted in Fig. 1A.

\section{Experimental: Stimulation frequency response functions}

The average \pm standard error baseline firing rates for Vim, Rt, STN, and SNr neurons were $32.0 \pm 11 \mathrm{~Hz}$, $8.2 \pm 1 \mathrm{~Hz}, 39.9 \pm 3 \mathrm{~Hz}$, and $102.3 \pm 16 \mathrm{~Hz}$, respectively. The stimulation frequency response functions (Fig. 1B) show excitatory responses for Vim and Rt, and inhibitory responses for STN and SNr. For Vim, neuronal firing rates progressively increased as the stimulation frequency became greater and a significant main effect of stimulation was found $\left[F=43.074(9,234), p<.001, \eta^{2}=0.624\right]$. Bonferroni-corrected t-tests revealed differences in neuronal firing compared to baseline at stimulation frequencies of $10 \mathrm{~Hz}(\mathrm{p}=.038)$, $30 \mathrm{~Hz}(\mathrm{p}=.041)$, and greater $(\mathrm{p}<0.05)$. For Rt, neuronal firing rates also progressively increased as the stimulation frequency became greater and a significant main effect of stimulation was found $[\mathrm{F}=31.170$ $\left.(9,117), p<.001, \eta^{2}=0.706\right]$. Statistically significant differences in neuronal firing compared to baseline were found at stimulation frequencies of $30 \mathrm{~Hz}(\mathrm{p}=.029)$ and greater $(\mathrm{p}<.05)$. For STN, neuronal firing rates were progressively attenuated as the stimulation frequency became greater and a significant main effect of stimulation was found $\left[F=26.420(9,91), p<.001, \eta^{2}=0.746\right]$. Statistically significant differences in neuronal firing compared to baseline were found at stimulation frequencies of $20 \mathrm{~Hz}(\mathrm{p}=.029)$ and greater $(\mathrm{p}<.001)$. For $\mathrm{SNr}$, neuronal firing rates also progressively attenuated as the stimulation frequency became greater and a significant main effect of stimulation was found $[\mathrm{F}=25.890(9,63), \mathrm{p}<.001$, $\left.\eta^{2}=0.787\right]$. Statistically significant differences in neuronal firing compared to baseline were found at stimulation frequencies of $3 \mathrm{~Hz}(\mathrm{p}<.05)$ and greater $(\mathrm{p} \leq .01)$. Detailed post hoc t-test statistics (all corrected for multiple comparisons within the text and figures) and Cohen's $d_{z}$ effect sizes are depicted in Fig. 1B. 


\section{Experimental: Time-domain responses to stimulation}

In Vim and Rt, periodic excitatory responses were evident at $5 \mathrm{~Hz}$ and $10 \mathrm{~Hz}$ (Fig. 2). The strength of the excitatory responses attenuated over time during stimulation trains of $\geq 20 \mathrm{~Hz}$ and were modelled by double exponential decay functions $\left(\mathrm{R}^{2}\right.$ values within Fig. 2). The time-series histograms for long train $\geq 100 \mathrm{~Hz}$ data ( $\geq 2 \mathrm{~s})$ in Vim and Rt show particularly prominent time-varying responses. While these stimulations did elicit excitatory responses, they were transient in nature and limited to start of stimulation. In Vim, the initial excitatory response at $200 \mathrm{~Hz}$ was of shorter duration than at $100 \mathrm{~Hz}$, and the subsequent neuronal suppressive response was stronger at $200 \mathrm{~Hz}$ than at $100 \mathrm{~Hz}$, with firing rates dropping below baseline. In $\mathrm{SNr}$, periodic inhibitory responses were evident at $5 \mathrm{~Hz}$ and $10 \mathrm{~Hz}$. In STN and $\mathrm{SNr}$, there was an overall stationary neuronal suppressive effect with increasing frequency (rather than an effect which changed dynamically over time as was the case in Vim and Rt).

\section{Computational: Responses to single stimulation pulses}

The net changes to postsynaptic currents in response to single pulses of stimulation were modelled by simultaneous activations of all presynaptic inputs (Fig. 3Bi). These responses differed across brain regions due to differences in the proportions of excitatory and inhibitory inputs (summarized in the Methods subsection "Computational: Presynaptic inputs" and Supplementary Table 1). The simulated peristimulus firing rate histograms (i.e. the neuronal responses to the aforementioned changes to presynaptic currents) revealed stimulus-evoked excitatory responses for Vim (peak firing rate of $405.9 \mathrm{~Hz} \mathrm{vs} .245 .1 \mathrm{~Hz}$ in the experimental data), inhibitory responses for $\mathrm{SNr}$ (minimum firing rate of $0 \mathrm{~Hz} \mathrm{vs} .0 .7 \mathrm{~Hz}$ in the experimental data), and a short-latency excitatory responses $(78.8 \mathrm{~Hz}$ peak vs. no peak in the experimental data) followed by a longer latency inhibitory response $(8.4 \mathrm{~Hz}$ trough vs. $4.8 \mathrm{~Hz}$ in the experimental data) for STN (Fig. 3Bii). The lack of short-latency excitation in the experimental data for STN might be explained by discrepancies in the temporal dynamics of excitatory transmission and/or occlusion of the excitatory response by the stimulus artifact. Figure generation for model Rt neurons was omitted due to redundancy as the model parameters are identical to Vim except for the parameter which underlies the baseline firing rate (elaborated upon in the Methods secion; model parameters for Rt are nevertheless provided in the Supplementary Material).

Computational: Time-domain synaptic currents 
Excitatory and inhibitory synaptic currents were generated separately, along with the total (i.e. sum of excitatory and inhibitory) synaptic currents in responses to DBS pulses across a range of frequencies for each of Vim, STN, and SNr (Fig. 4). The TM model accounts for frequency-dependent changes to shortterm synaptic dynamics. In all structures, the model suggests non-selective frequency-dependent depression of both excitatory and inhibitory synaptic currents. For Vim, sustained periodic excitations are seen with $5 \mathrm{~Hz}$ and $10 \mathrm{~Hz}$, while frequency-dependent weakening of the excitatory responses with successive stimuli are observed with frequencies $\geq 20 \mathrm{~Hz}$. Predominant inhibitory synaptic currents corroborate the strong inhibitions of somatic firing in SNr with low stimulation frequencies; whereas neuronal suppression with higher frequencies is likely the result of non-selective frequency-dependent synaptic depression. For STN, the mixed excitatory-inhibitory stimulus-evoked responses likely explain the more net-neutral somatic firing responses in experimental data with lower stimulation frequencies; whereas non-selective synaptic depression can explain the frequency-dependent suppression of somatic firing with higher stimulation frequencies.

\section{Computational: Time-domain membrane potentials}

The membrane potentials of modelled neurons in response to DBS across a range of frequencies were generated for each of Vim (Fig. 5), STN (Fig. 6), and SNr (Fig. 7). The proportions of excitatory and inhibitory inputs (Supplementary Table 1) together with the parameters of the model neurons (Supplementary Table 2) generated baseline (DBS-OFF) firing rates which corresponded to in vivo recordings. The left side of Fig. 5 shows the simulated membrane potential (accounting also for action potential generation) before and during stimulation across a range of frequencies for Vim, whereas the right side shows an exemplary in vivo Vim neuron. A selective oscillation due to stimulus entrainment is reproduced by the model neuron for DBS at $20 \mathrm{~Hz}$. The model neuron can moreover partially reproduce the transient excitatory responses at DBS onset with $50 \mathrm{~Hz}$ and $100 \mathrm{~Hz}$ stimulation and $30 \mathrm{~Hz}$ to some degree; however the transient excitatory responses within the model are of shorter latency. For STN (Fig. 6 ), the simulated (left) neuronal firing compared to baseline decreases for DBS at $\geq 30 \mathrm{~Hz}$, corroborating experimental data (exemplary in vivo STN neuron portrayed on the right side). Neuronal firing rates are substantially attenuated with DBS at $50 \mathrm{~Hz}$ and $100 \mathrm{~Hz}$ (as is the case experimentally) due to non-selective synaptic depression. For SNr (Fig. 7), the simulated (left) neuronal firing rate decreases dramatically beginning at $20 \mathrm{~Hz}$ due to the dominant inhibitory presynaptic currents, corroborating experimental data (exemplary in vivo $\mathrm{SNr}$ neuron portrayed on the right side). The model neuron fails to generate action 
potentials for DBS $\geq 50 \mathrm{~Hz}$ (as is the case experimentally) due to non-selective synaptic depression. Timedomain histograms are also presented in each figure (Fig. 5, 6, 7) which were generated by averaging the neuronal firing rates of 10 modelled neurons for each respective structure across $2 \mathrm{~s}$ of stimulation at each frequency. 


\section{Discussion}

\section{Site-specific and frequency-dependent stimulation effects}

At the somatic level, electrical stimulation is both site-specific and frequency-dependent. In Vim and Rt, neuronal activity could be upregulated, whereas in STN and SNr it was downregulated. These mechanistic disparities across brain regions are most likely explained by anatomical differences in local microcircuitries, in that the effects appeared dependent upon the relative distributions of excitatory and inhibitory inputs converging at target neurons (Chiken and Nambu, 2014). The experimental findings demonstrated that neuronal activity in any brain region could be suppressed either selectively in regions with a high predominance of inhibitory inputs or non-selectively if high enough stimulation frequencies were used. Neuronal excitation, however, could only be achieved when electrical stimulation was delivered to brain regions with a high predominance of glutamatergic inputs. While these bimodal effects (excitatory vs. inhibitory) with low stimulation frequencies were likely attributable to presynaptic activation, the loss of site-specificity and convergence towards neuronal suppression with sustained HFS $(\geq 100 \mathrm{~Hz}$ ) was most likely attributable to synaptic depression (Liu et al., 2012; Milosevic et al., 2018a; Rosenbaum et al., 2014; Steiner et al., 2019). This phenomenon of short-term synaptic plasticity can be defined as a reversible decrease in synaptic efficacy, caused by the depletion of readily releasable neurotransmitter vesicle pools when successive stimuli are delivered at a fast ratel; a reduction of presynaptic calcium conductance; and/or the inactivation of neurotransmitter release sites due to delayed recovery from vesicle fusion events (Dittman and Regehr, 1998; Fioravante and Regehr, 2011; Rizzoli and Betz, 2005; Rosenmund and Stevens, 1996; Zucker and Regehr, 2002).

Our computational model was designed to test our two main hypotheses, (i) that the post-synaptic responses (i.e. neuronal output) to single pulses of electrical stimulation were mediated by the proportions of inhibitory vs. excitatory inputs to the stimulated neuron, and (ii) that weakened synaptic transmission fidelity over time with higher stimulation frequencies was mediated by short-term synaptic plasticity. As such, the biophysical modelling approach takes into consideration both anatomical (local microcircuitry) and physiological (short-term synaptic dynamics) properties. At stimulation frequencies below the threshold for synaptic depression (i.e. $<20-30 \mathrm{~Hz}$ ) (Liu et al., 2012; Milosevic et al., 2018a), our model showed that neuronal responses were the result of a temporal summation of stimulus-evoked responses. In structures with predominantly excitatory inputs, this led to increases in neuronal output, whereas the opposite occurred in structures with predominantly inhibitory inputs. Beyond the threshold for synaptic 
depression, the strengths of successive stimulus-evoked responses were progressively reduced (i.e. a loss of synaptic transmission fidelity). In the Vim, with high frequencies, we observed an initial excitatory response which weakened over time. The $\mathrm{SNr}$ too was affected by synaptic depression, although the impact upon neuronal firing was unable to be observed since the stimulus-evoked inhibitory responses are so strong that neuronal silencing can be achieved by temporal summation of inhibitory events alone (thus one cannot observe the neuronal output). However, we have previously shown progressive, frequencydependent decreases to the amplitudes of extracellular evoked field potentials in $\mathrm{SNr}$ with stimulation frequencies $\geq 20 \mathrm{~Hz}$ (Milosevic et al., 2018a). It may then be assumed that since synaptic depression would weaken the strength of inhibitory synaptic transmission, neuronal firing should increase via disinhibition. However, our model shows non-selective synaptic depression of both inhibitory and excitatory synaptic currents. This is indeed supported by experimental work in rodent STN slices which demonstrated that pharmacologically-isolated excitatory and inhibitory postsynaptic potentials were both depressed during HFS (Steiner et al., 2019). High-frequency DBS has therefore been considered a "functional deafferentation" (Anderson et al., 2004). This would also explain the suppression of somatic firing in STN with higher stimulation frequencies, whereas the stimulus-evoked responses with lower frequencies produced rather weak net inhibitory responses due to the more homogenous distribution of excitatory and inhibitory inputs to STN.

\section{Stimulation of the basal ganglia}

Individual stimulation pulses elicited net inhibitory responses in both STN and SNr. These responses are corroborated by anatomical studies which suggest a predominance of GABAergic inputs to each of these structures (summarized in the STAR Methods subsection "Computational: Presynaptic inputs"). The greater predominance of inhibitory inputs to $\mathrm{SNr}$ thus explains the stronger inhibitory responses compared to STN. While single pulses of stimulation at low frequencies elicited inhibitory responses, with higher stimulation frequencies (i.e. $\geq 20 \mathrm{~Hz}$ ), neuronal inhibition/suppression was even more easily achieved likely due to a combined effect of inhibitory temporal summation and non-selective synaptic depression.

The canonical model of Parkinson's disease suggests that the activity of the STN is pathologically increased as a result of a lack of downregulation of D2-dopamine-receptor-mediated "indirect-pathway" projections (Albin et al., 1989; Alexander and Crutcher, 1990; DeLong, 1990; Surmeier et al., 2007). The subsequent increased excitatory drive of subthalamo- pallidal and nigral projections, in combination with a lack of upregulation of D1-dopamine-receptor-mediated "direct-pathway" projections, leads to a 
pathological overactivity of the GPi and SNr. As such, in Parkinson's disease, the STN, SNr, and GPi may all benefit from the suppression of neuronal overactivity, and/or stimulation-induced abolishment of pathophysiological oscillations (Kühn et al., 2008) which manifest throughout the disease process (Brown, 2003). It must also, however, be considered that while somatic firing would be suppressed in these structures, each DBS pulse may initiate an action potential along the efferent axons (McIntyre et al., 2004). Thus, high-frequency DBS may work to replace pathological somatic firing with regularized highfrequency output.

\section{Stimulation of the thalamic ventral intermediate nucleus}

In the Vim, individual stimulation pulses elicited time-locked excitatory responses. While stimulusevoked inhibitory responses in SNr and STN were likely caused by the predominance of inhibitory afferent inputs, the excitatory responses elicited in Vim were most likely explained by the predominant excitatory afferent inputs. In high-frequency stimulation of the Vim, somatic firing was transiently upregulated at the start of stimulation, but this effect weakened quickly and progressively over time. As our model demonstrates, the initial excitatory response can likely be explained by synaptic activation of the predominant glutamatergic afferents, while the subsequent weakening is likely the result of synaptic depression (Ran et al., 2009) due to the fast rate at which subsequent stimuli are delivered. In an intracellular sensorimotor thalamic rat brain slice, it was indeed shown that $125 \mathrm{~Hz}$ stimulation resulted in an initial depolarization response coupled with increased neuronal firing, followed quickly by (complete or incomplete) repolarization coupled with suppression of neuronal firing (Anderson et al., 2004). These responses were reversed by application of glutamate receptor and calcium channel antagonists, demonstrating their presynaptic nature. The neuronal suppression which occurred after the initial excitation was indeed suggested to be the result of synaptic depression.

The pathology of essential tremor is not well understood, but is increasingly being studied, with several lines of evidence indicating a dysfunction and possible degeneration of the cerebellum (Louis et al., 2007, 2019); which subsequently projects to the Vim. Postmortem literature has revealed pathologies including but not limited to Purkinje cell loss (Axelrad et al., 2008; Shill et al., 2008), Purkinje cell axonal and dendritic swelling (Louis et al., 2011; Yu et al., 2012), and reduced cerebellar GABAergic tone (ParisRobidas et al., 2012). These pathological changes may indeed drive the characteristic neurophysiological changes that occur in patients with essential tremor (Helmich et al., 2013), including tremor-related oscillations at the local field potential (Kane et al., 2009) and single-unit (Lenz et al., 1988) levels which 
are coherent with tremor at the periphery. As such, the therapeutic efficacy of high-frequency Vim-DBS might be explained by suppression of neuronal firing and/or abolishment of pathophysiological tremorrelated oscillatory activity (Milosevic et al., 2018b). Conversely, low-frequency stimulation-induced tremor (Barnikol et al., 2008; Swan et al., 2016) might explained by periodic neuronal entrainment which mimics tremor-related neuronal activity.

\section{Stimulation of the reticular thalamus}

In the Rt, like in the Vim, individual stimulation pulses elicited time-locked excitatory responses. Also like in the Vim, the upregulation of neuronal firing during stimulation at $\geq 20 \mathrm{~Hz}$ weakened over time, and with $100 \mathrm{~Hz}$ stimulation, the excitatory responses were limited to the first $\sim 0.5 \mathrm{~s}$ of the stimulation train, after which somatic firing was reduced to or below baseline. A recent in vivo nonhuman primate study (Lymer et al., 2019) demonstrated inhibitory responses which followed $0.5 \mathrm{~s}$ trains of microstimulation at $100 \mathrm{~Hz}$ (note: neuronal firing was not assessed during stimulation) hypothesized to have been mediated by GABAergic afferent inputs to the Rt. Here, we showed that $0.5 \mathrm{~s} 100 \mathrm{~Hz}$ stimulation trains in fact initially drove neuronal firing during stimulation, which likely reflects the prominent glutamatergic input, after which neuronal firing was suppressed likely due to synaptic depression. Slice work has indeed demonstrated that electrical stimulation of the Rt can elicit EPSCs (Ulrich and Huguenard, 1995) and neuronal spiking (Landisman et al., 2002; Schlag and Waszak, 1971). Moreover, stimulation of layer VI cortical neurons (Gentet and Ulrich, 2004), and ventrobasal (Gentet and Ulrich, 2003) and ventrolateral (Bazhenov et al., 1999) thalamic neurons elicited excitatory responses in Rt.

A conventional application of Rt-DBS does not exist; however, in animal studies, Rt stimulation has been implicated as a possible treatment for various forms of epilepsy (Chang et al., 2017; Nanobashvili et al., 2003, 2012; Pantoja-Jiménez et al., 2014; Wang and Wang, 2017). Hypersynchronization of Rt output has been implicated in spike-wave seizures (Blumenfeld, 2005), supporting a rationale for Rt-DBS for intractable epilepsy (Krauss and Koubeissi, 2007). Moreover, the GABAergic output of the Rt has an important role in controlling excitability throughout the thalamus as it is known to project to many different thalamic nuclei (Cox et al., 1997; Kim et al., 1997; Kultas-Ilinsky et al., 1995; Steriade et al., 1984). In experimental applications of Rt-DBS, it must be considered that upregulation of Rt neuronal activity would have a widespread inhibitory effect on downstream thalamic structures, while suppression of Rt activity would lead to disinhibition (unless its output is indeed replaced by regularized DBS pulses). While not specifically applied to Rt, DBS of other nodes of the ascending reticular activating system have 
also been investigated for minimally conscious state (Chudy et al., 2018; Yamamoto and Katayama, 2005) due to the role of this system in behavioural arousal and consciousness. Moreover, stimulation of the pontomesencephalic tegmentum area has been shown to modulate sleep in patients with Parkinson's disease (Arnulf et al., 2010; Lim et al., 2009). Considering the role of Rt in arousal and attention (Lewis et al., 2015; McAlonan et al., 2008) and the role of synchronous Rt neuronal activity (which can be induced by stimulation as seen in Supplementary Fig. 2) during sleep (Landisman et al., 2002), stimulation of the Rt may have a possible role in sleep disorders.

\section{Translational implications}

The selectively bimodal and frequency-dependent somatic responses described here should be taken into consideration in the development of novel stimulation paradigms and DBS indications. In applications of DBS which utilize a high stimulation frequency, suppression of somatic firing is likely achieved. Stimulation paradigms which utilize low stimulation frequencies and are applied to areas of the brain with predominantly glutamatergic inputs may depend upon periodic facilitation of somatic firing, with one possible example being low-frequency pedunculopontine-DBS (Moreau et al., 2009). Low-frequency stimulation in an area of the brain with predominantly inhibitory inputs may on the other hand cause periodic inhibitions. In either case, low-frequency stimulation can induce oscillatory neuronal behaviour (as seen in Supplementary Fig. 2). Knowledge of the site-specific and frequency-dependent properties of DBS can inform the development of novel stimulation paradigms such as closed-loop stimulation for on demand upregulation or downregulation of neuronal firing, or for induction or disruption of neuronal oscillations. Indeed, stimulation parameters are often decided upon empirically. Based on the findings presented here, knowledge of the local microcircuitry (distribution of afferent inputs) inherent to the stimulated brain region (i.e. therapeutic targets of interest for DBS application) may allow us to infer/predict the stimulation frequency response properties. As such, our comprehensive computational model may represent a valuable tool for physiologically-informed stimulation programming and paradigm development in prospective DBS targets and indications, particularly as our model was developed based on in vivo experimental data from the human brain.

\section{Considerations and limitations}

While suppression of somatic activity may indeed be therapeutic (Bergman et al., 1990; Levy et al., 2001; Wichmann et al., 1994), orthodromic and antidromic axonal effects of electrical stimulation must also be considered (McIntyre and Hahn, 2010). If each DBS pulse generates an axonal action potential (McIntyre 
et al., 2004), then the overall "neuronal output" should be considered as the summation of the somatic firing rate (which would have been influenced by afferent axon activations) and stimulation frequency (i.e. efferent axon activation); this summation is incorporated into Fig. 1B. Thus, HFS applications which completely suppresses somatic firing would replace neuronal output with regular outputs corresponding to the stimulation frequency. Although we did not record from any structures downstream of the stimulation site, it is perhaps possible to infer the downstream effects based on the results presented here. For example, in stimulation of the STN, activation of the glutamatergic subthalamo-pallidal/nigral efferents may cause excitatory responses downstream (Boulet et al., 2006; Galati et al., 2006; Hashimoto et al., 2003), especially if lower stimulation frequencies are used; whereas with higher stimulation frequencies the downstream glutamatergic drive may in fact be weakened (Maltête et al., 2007; Tai et al., 2003; Zheng et al., 2011) due to synaptic depression. Further studies are warranted in order to better understand the possible orthodromic (and antidromic) network phenomena of DBS (Alhourani et al., 2015). Moreover, studies relating to the downstream and upstream DBS effects would allow us to better understand the mechanisms of DBS applied to white matter tracts (such as forniceal-DBS). Another notable limitation of this study is that the applied stimulation trains were limited to short durations; stimulation effects over longer durations are yet to be validated. Moreover, while this study aimed to elucidate differential mechanisms involved in stimulation of various brain structures, behavioural and clinical correlates were not assessed here directly. However, the high-frequency microstimulation applied to Vim was shown to be effective at suppressing tremor (Milosevic et al., 2018b) confirming that the stimulation parameters used were clinically relevant. Moreover, the stimulation parameters used here (in particular, the $100 \mathrm{~Hz}$ microstimulation trains) are comparable in terms of total electrical energy delivered during clinically-applied DBS macrostimulation (Milosevic et al., 2019) though are of greater current density due to the smaller stimulating surface. Finally, an important limitation of this work is that the explanation of site-specific mechanistic disparities based on the proportions of inhibitory/excitatory afferent inputs does not account for the possible contributions of glia (Bekar et al., 2008; Campos et al., 2020; Salatino et al., 2017; Tawfik et al., 2010) nor neuromodulatory inputs (Lavian et al., 2018; Lavoie et al., 1989) which should be considered in future work.

\section{Conclusion}

The presented results demonstrate the site-specific and frequency-dependent neuronal effects of extracellular stimulation. Neuronal suppression could be achieved either by stimulus-evoked inhibitory 
events in structures with predominantly GABAergic inputs (STN and SNr) or non-selectively when sustained HFS was delivered. Stimulus-evoked neuronal excitatory responses were exclusive to structures with predominantly glutamatergic inputs (Vim and Rt), particularly with lower stimulation frequencies. Our computational model showed that the bimodal site-specific stimulus-evoked responses could be explained by differences in the distributions of inhibitory and excitatory inputs to the stimulated target structures, whereas convergence towards neuronal suppression with sustained HFS could be explained by synaptic depression. 


\section{A Peristimulus responses to single stimulation pulses}
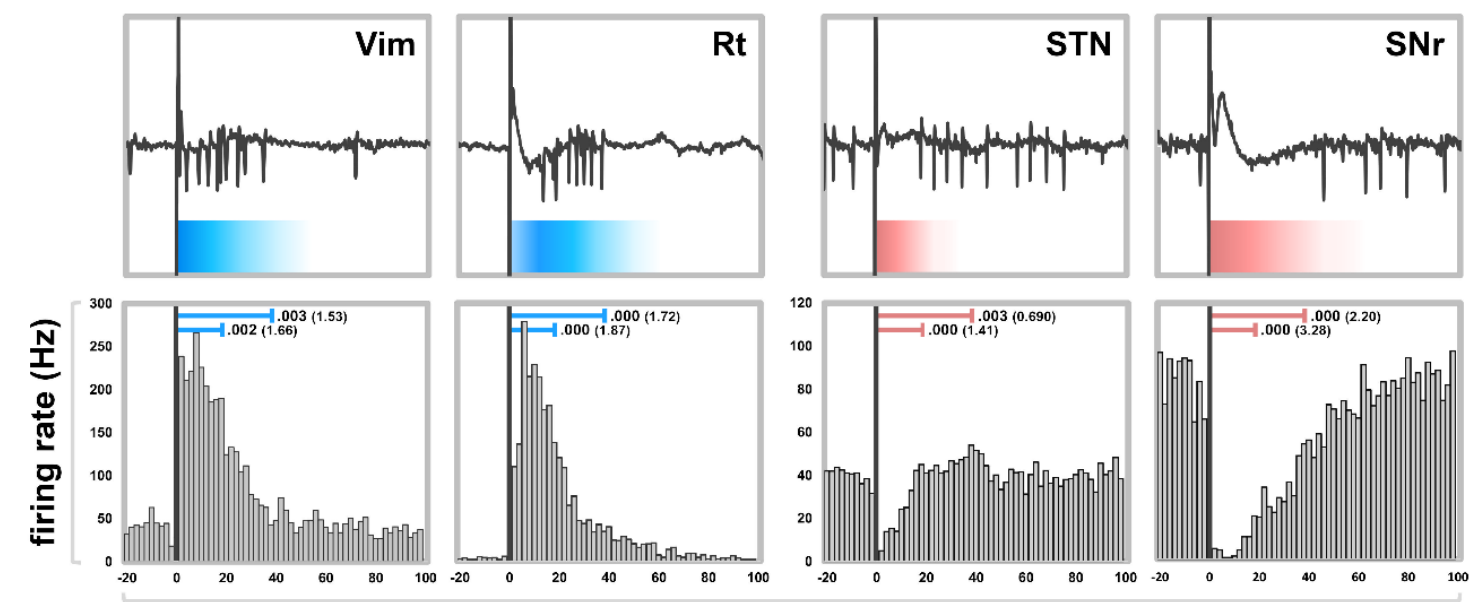

time (ms)

\section{B Frequency responses ( $\leq 60$ pulses)}

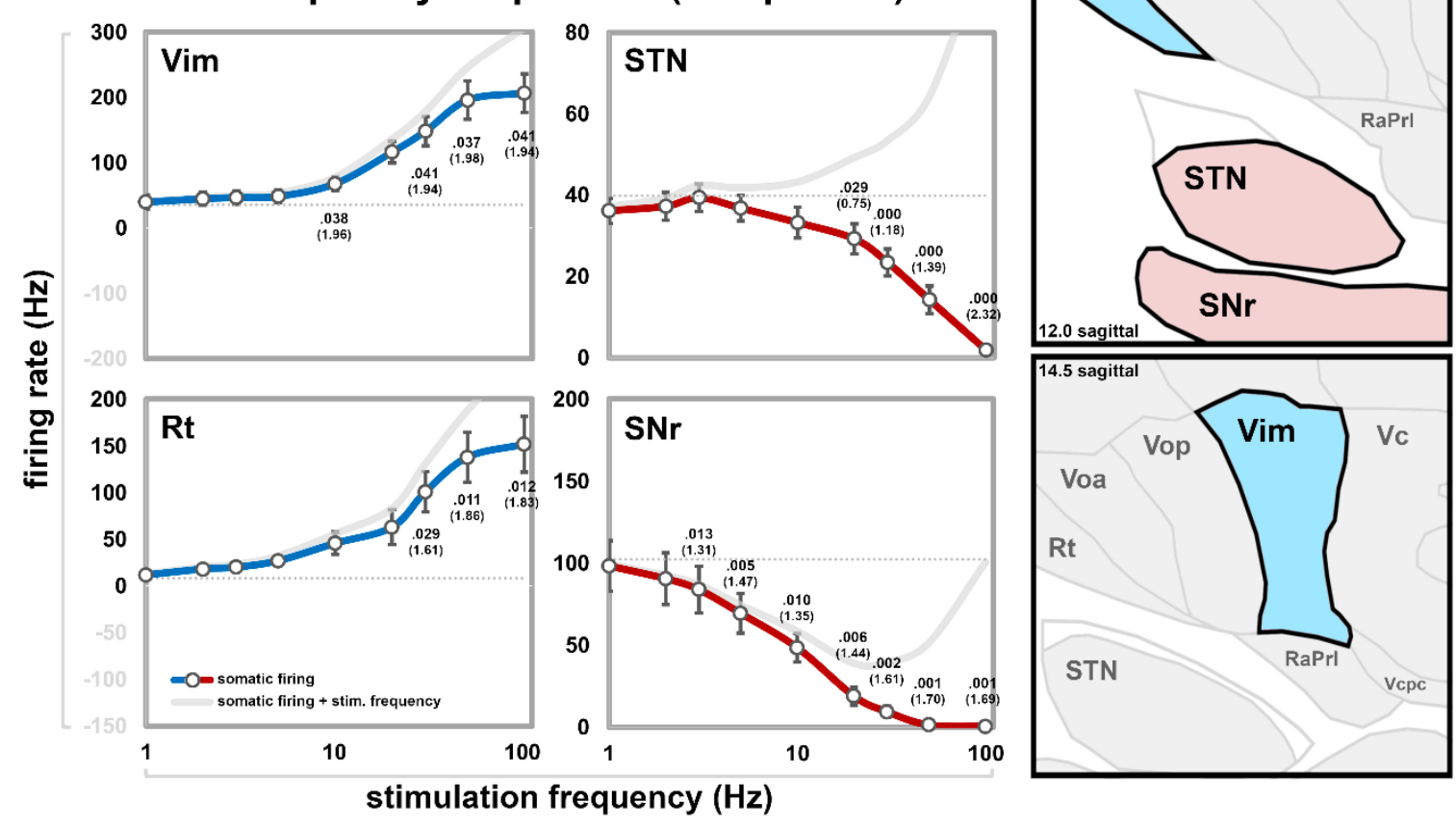

Fig. 1 - Experimental (A) peristimulus responses and (B) frequency response functions. (A) Top panels show exemplary responses to a single stimulation pulse in each structure, whereas bottom panels show groupwise peristimulus time histograms of stimulus-evoked excitatory responses for Vim and Rt and stimulus-evoked inhibitory responses for STN and SNr. The average firing rates of the immediate $20 \mathrm{~ms}$ and $40 \mathrm{~ms}$ periods following stimulations pulses were significantly greater than the 20ms pre-stimulus periods for Vim and Rt, and significantly lesser for STN and SNr (Bonferonni-corrected 2-tailed paired ttests, Cohen's $d_{z}$ effect sizes in parentheses). (B) Stimulation ( $\leq 60$ pulses) frequency response functions show that average firing rates progressively increased in Vim and Rt as the stimulation frequency became greater, while they progressively decreased in STN and SNr. The average \pm standard error baseline firing rates for Vim, Rt, STN, and SNr neurons were $32.0 \pm 11 \mathrm{~Hz}, 8.2 \pm 1 \mathrm{~Hz}, 39.9 \pm 3 \mathrm{~Hz}$, and $102.3 \pm 16 \mathrm{~Hz}$, respectively (dashed gray lines). Firing rates during the various stimulation trains were compared to the baseline firing rates and the results of Bonferroni-corrected post hoc t-tests (2-tailed, paired) are displayed with Cohen's $d_{z}$ effect sizes in parentheses. In the case that each DBS pulse generates an action potential on the efferent axon, then the overall neuronal output would be the summation of the somatic firing rate and stimulation frequency; this is represented by the solid gray lines in each plot. The right anatomical panels are $12.0 \mathrm{~mm}$ and $14.5 \mathrm{~mm}$ sagittal sections. 


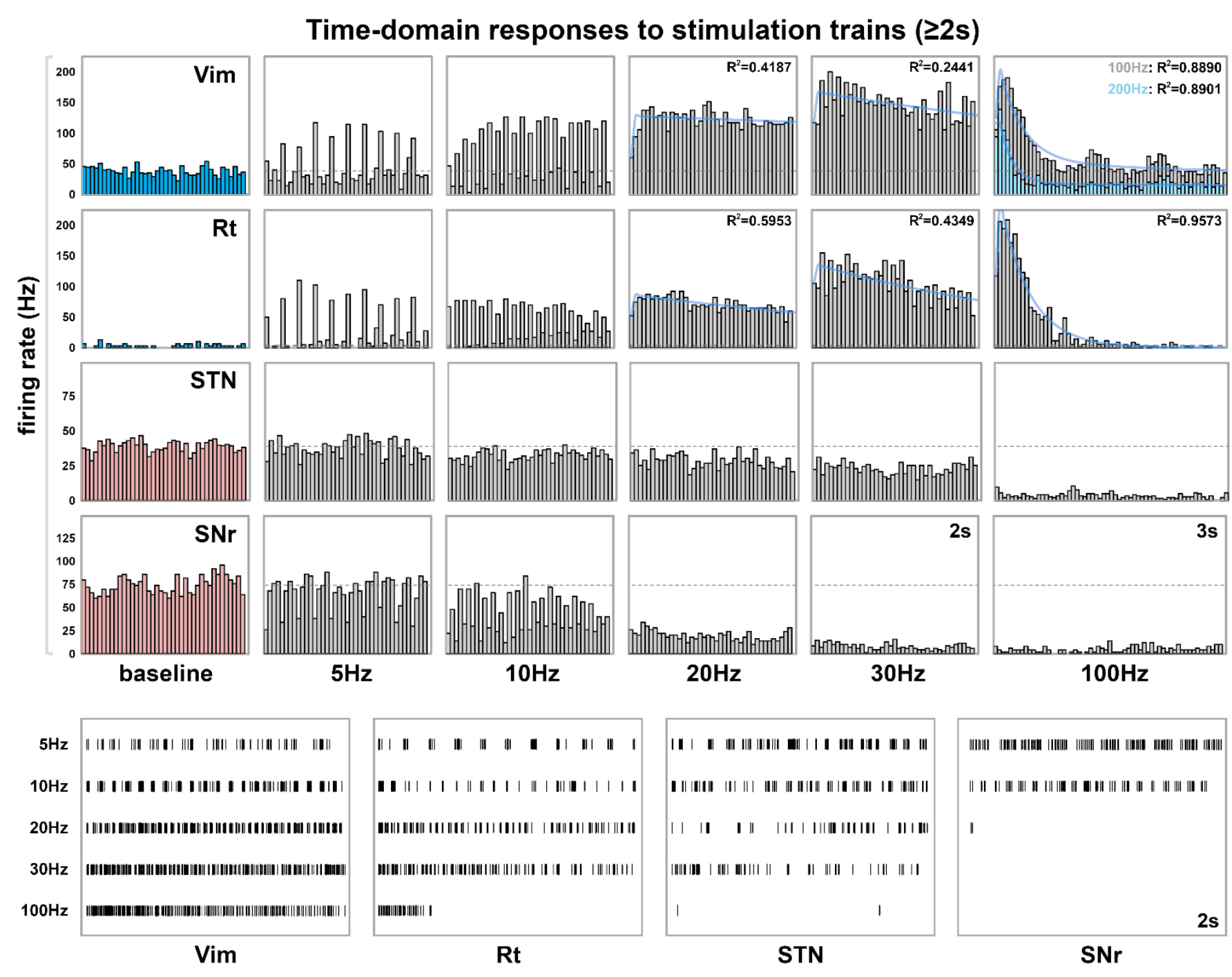

Fig. 2 - Experimental time-domain responses to stimulation trains. For Vim and Rt, firing rates progressively increased above baseline (gray dashed lines) with increasing stimulation frequencies. Periodic excitatory responses are shown at $5 \mathrm{~Hz}$ and $10 \mathrm{~Hz}$, however neuronal excitation declined over time with $\geq 20 \mathrm{~Hz}$. Excitatory responses with $100 \mathrm{~Hz}$ long trains $(\geq 2 \mathrm{~s}$ ) were transient, and a subsequent reduction of neuronal firing is evident after the initial excitation. In Vim, the initial excitatory response at $200 \mathrm{~Hz}$ is of shorter duration than at $100 \mathrm{~Hz}$, and the subsequent neuronal suppressive response is stronger at $200 \mathrm{~Hz}$ compared to $100 \mathrm{~Hz}$, with firing rates dropping below baseline. In STN and SNr, firing rates progressively decreased with increasing stimulation frequencies. In SNr, periodic inhibitory responses are visible at 5 and $10 \mathrm{~Hz}$. Exemplary firing rate raster data from each structure during various stimulation trains are displayed in the four bottom-most panels. 


\section{A Conceptual framework for modelling single pulse responses}

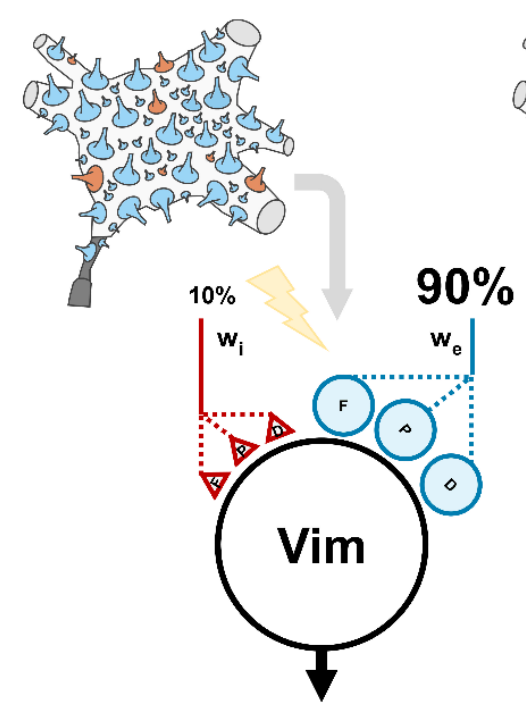

strong excitation

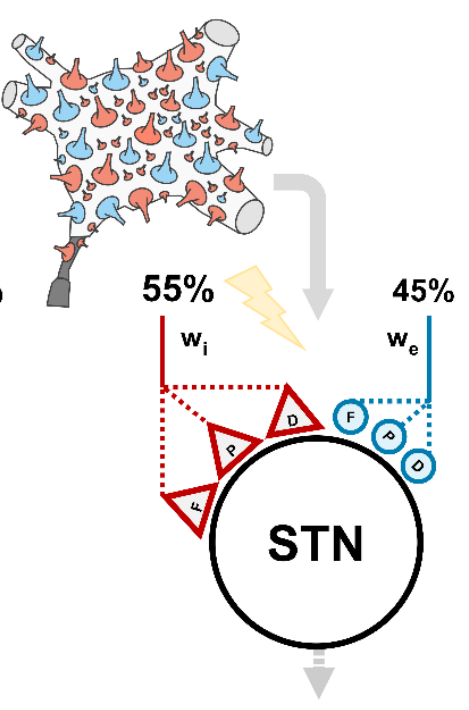

weak inhibition

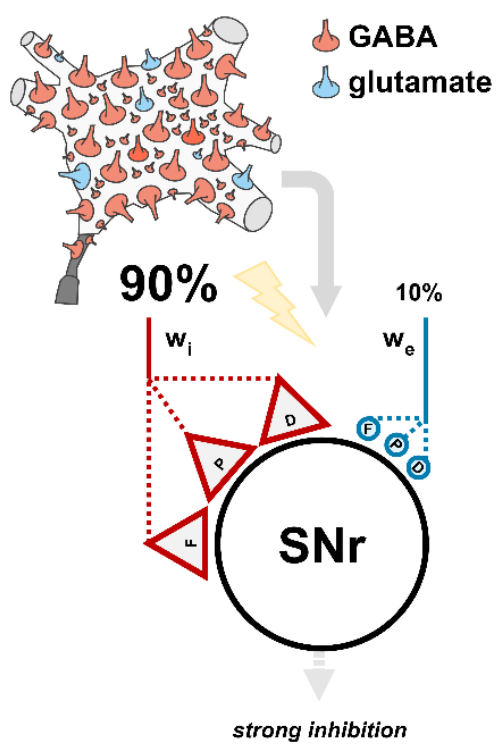

\section{B Simulated responses to single stimulation pulses}

(i) DBS-induced changes to synaptic currents via simultaneous activation of all afferent inputs
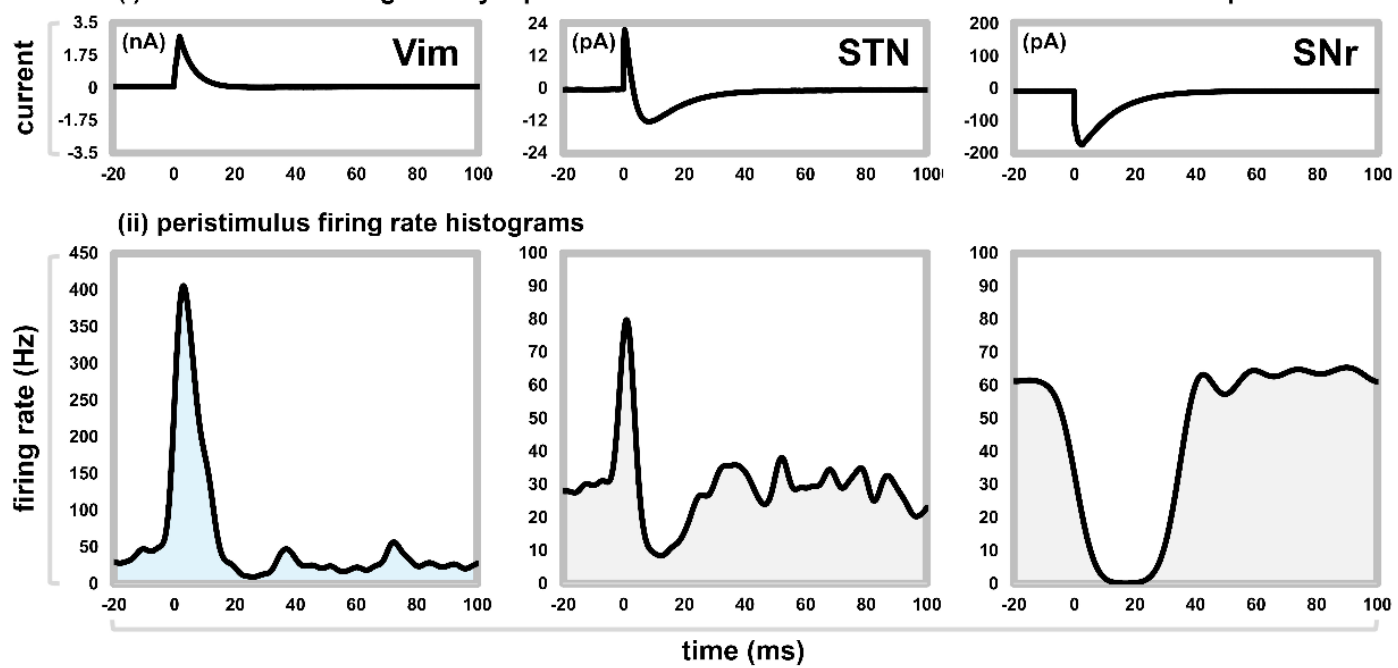

Fig. 3 - Computational (A) model framework and (B) simulated peristimulus responses. (A) To model the response to single pulses of electrical stimulation, each model neuron was assigned a certain proportion of excitatory and inhibitory presynaptic inputs/weights with proportions derived from anatomical literature. The effect of each DBS single pulse was modelled by simultaneously activating all presynaptic inputs. (B) The corresponding changes to (i) synaptic currents and (ii) somatic firing induced by the simultaneous activations are displayed (i.e. the singlepulse responses). This framework closely replicated the robust stimulus-evoked neuronal excitation in Vim and neuronal inhibition in SNr. In STN, there was a short-latency neuronal excitation which was not observed in the experimental data (though may have been occluded by the stimulation artifact) due to the high speed of excitatory synaptic transmission, followed by an inhibitory period congruent with the experimental data. 


\section{Simulated synaptic currents with short-term plasticity}
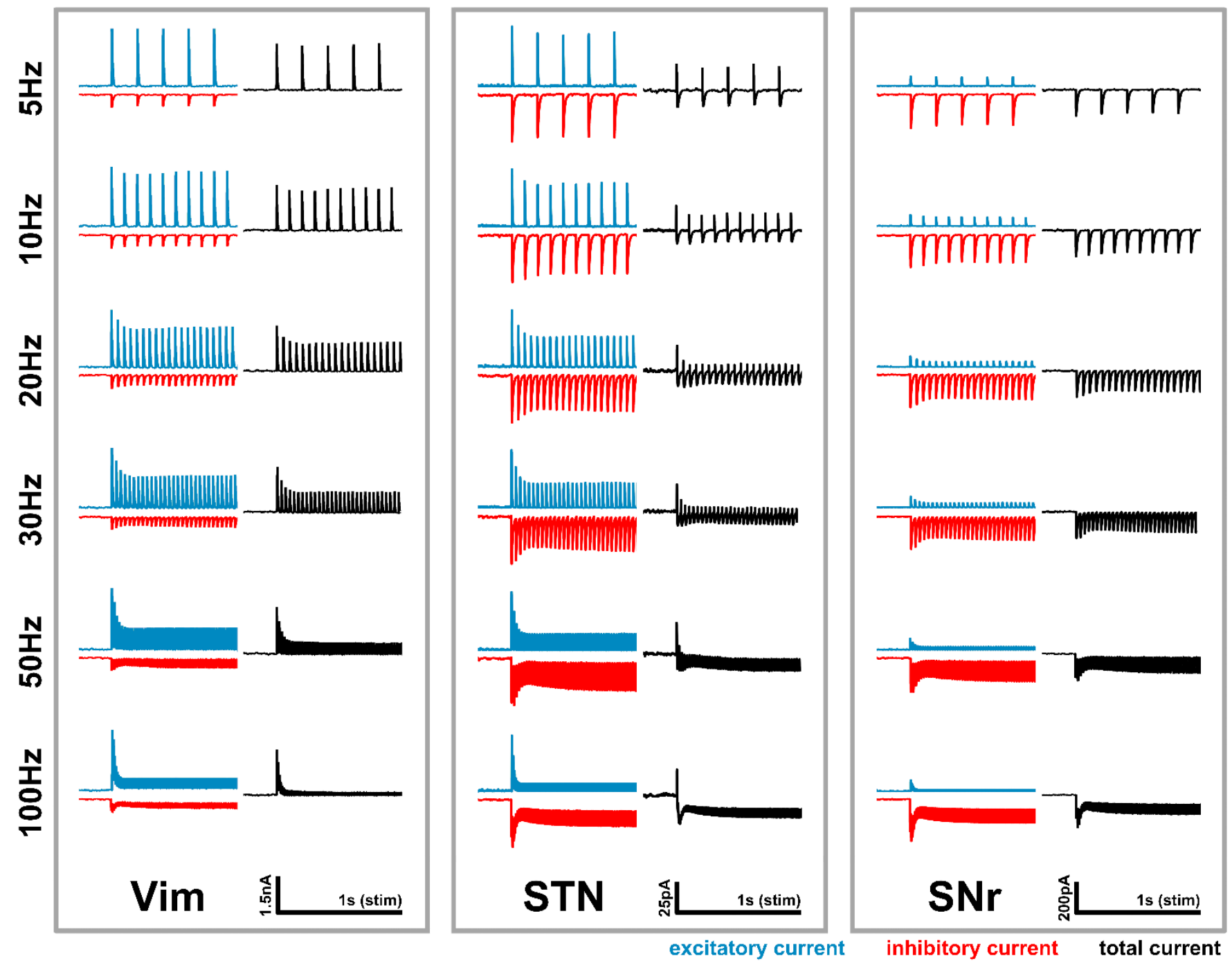

Fig. 4 - Computational time-domain synaptic currents. The three figures show excitatory, inhibitory, and total (i.e. sum of excitatory and inhibitory) synaptic currents in responses to DBS pulses across a range of frequencies with an embedded TM model to account for short-term synaptic dynamics. In all cases, the model suggests nonselective frequency-dependent depression of both excitatory and inhibitory synaptic currents. For Vim, rather stable periodic excitations are seen with $5 \mathrm{~Hz}$ and $10 \mathrm{~Hz}$. Also corroborating experimental data, frequency-dependent weakening of the excitatory responses is observed with frequencies $\geq 20 \mathrm{~Hz}$. Predominant inhibitory synaptic currents corroborate the strong inhibitions of somatic firing in $\mathrm{SNr}$, together with non-selective frequencydependent synaptic depression. For STN, the mixed excitatory-inhibitory stimulus-evoked responses likely explain the more net-neutral somatic firing responses in experimental data with lower stimulation frequencies, while nonselective synaptic depression can explain frequency-dependent suppression of somatic firing. 


\section{Vim - simulated and real neuronal activity}

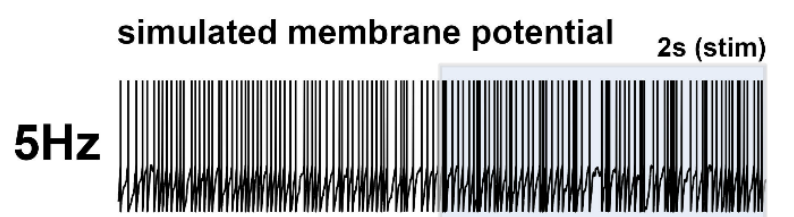

$10 \mathrm{~Hz}$
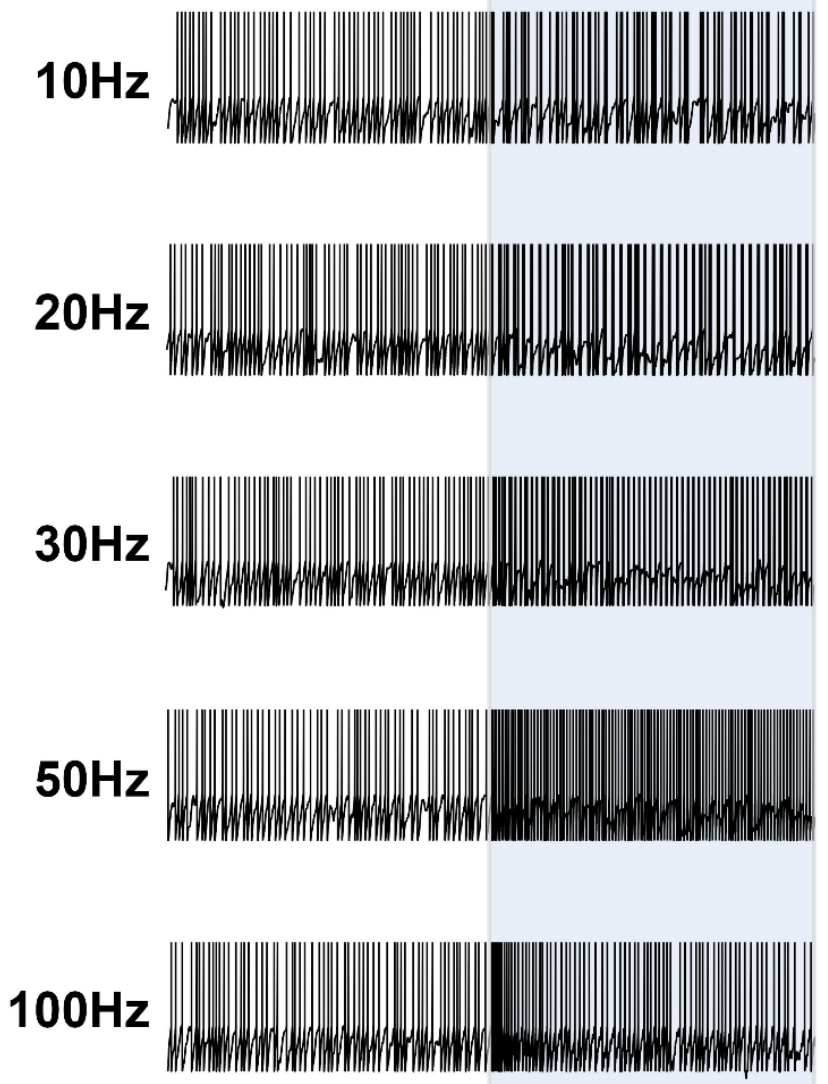

in vivo extracellular recording $2 \mathrm{~s}$ (stim)

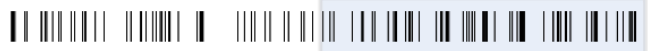

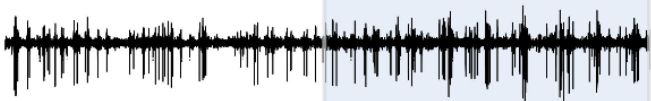

\section{|| || || ||||||| |||||| || ||||| || || || | | | || | | ||| |||||| | ||||||||| | |||||}

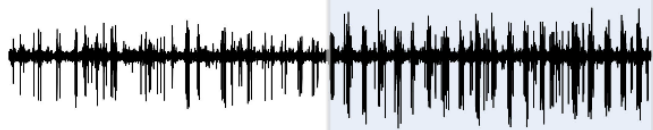

|||||||||| ||||||||||||||||||| |||||||||||||||||||||||||||||||||||||||||||||||||||||||||||||
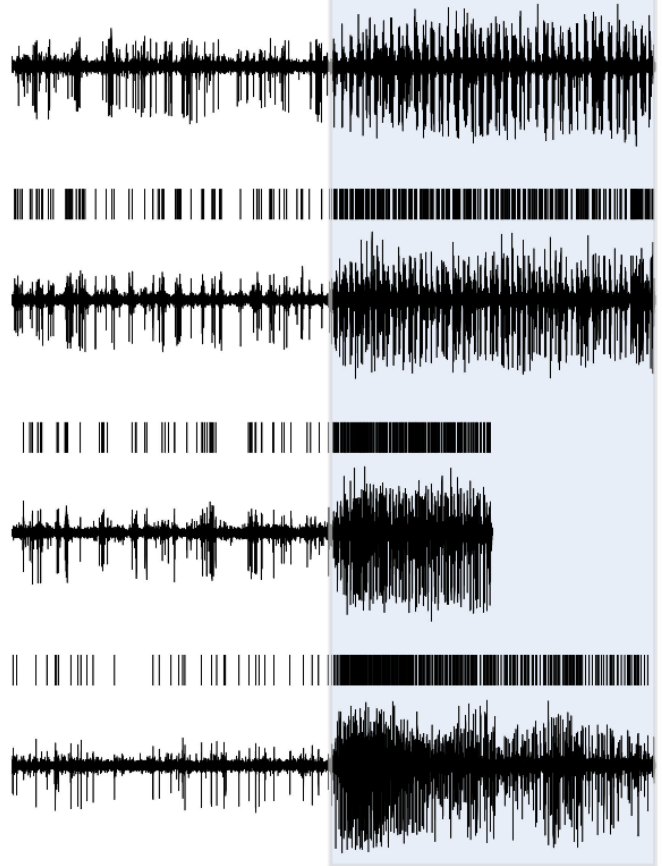

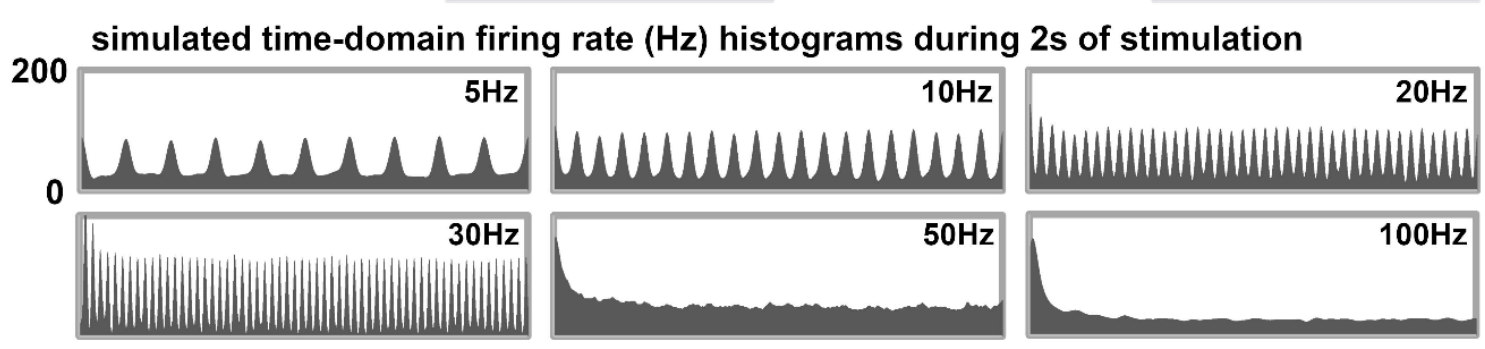

Fig. 5 - Computational time-domain membrane potential for Vim. The left panels show the membrane potential of a model Vim neuron immediately before (non-shaded) and during (shaded) DBS across a range of frequencies. The right panels are exemplary recordings from an in vivo human Vim neuron (stimulation for $50 \mathrm{~Hz}$ was limited to $1 \mathrm{~s})$. The bottom-most panels are time-domain firing rate histograms generated by averaging across 10 model Vim neurons. A selective oscillation due to stimulus entrainment is reproduced by the model neuron for DBS at $20 \mathrm{~Hz}$. The model neuron can partially generate the transient excitatory responses at DBS onset with $50 \mathrm{~Hz}$ and $100 \mathrm{~Hz}$ stimulation and $30 \mathrm{~Hz}$ to some degree; however, the transient excitatory responses within the model are of shorter latency. 


\section{STN - simulated and real neuronal activity}

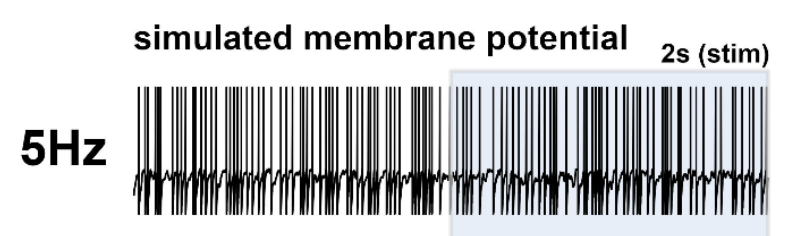

in vivo extracellular recording $2 \mathrm{~s}$ (stim)

|| || || || || || || || ||| || |||||| || || || | | || || ||||| || || | || || || | || ||

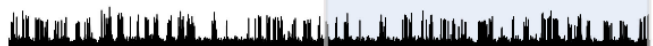

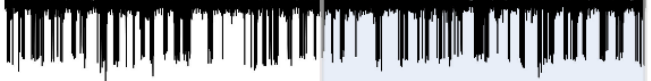

$10 \mathrm{~Hz}$

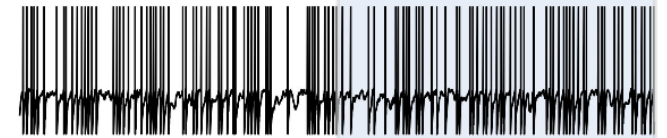

||| || || || || || ||| || ||||||| | | || || ||||| || || || || |||| || || ||| || ||||||

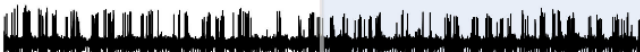

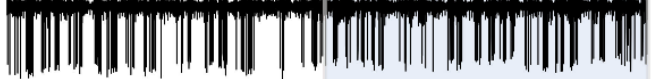

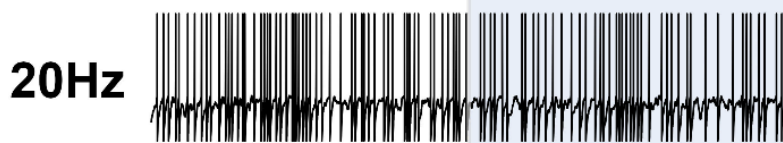

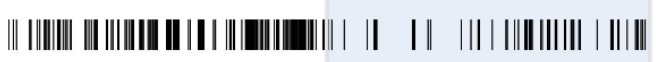

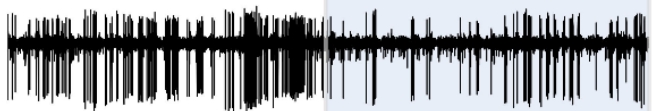
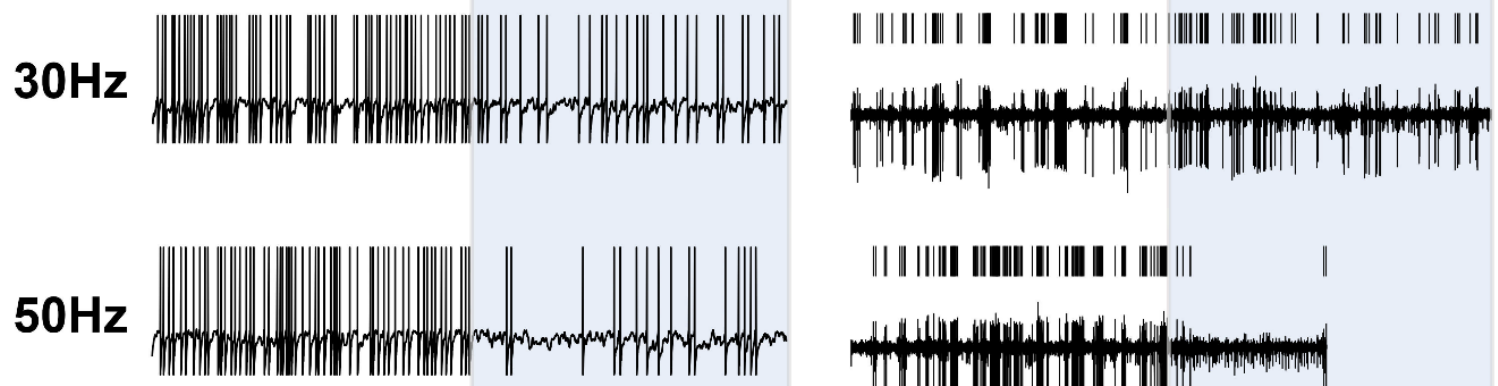

\section{|| || ||||| |||||| ||| |||| || || |||| ||}
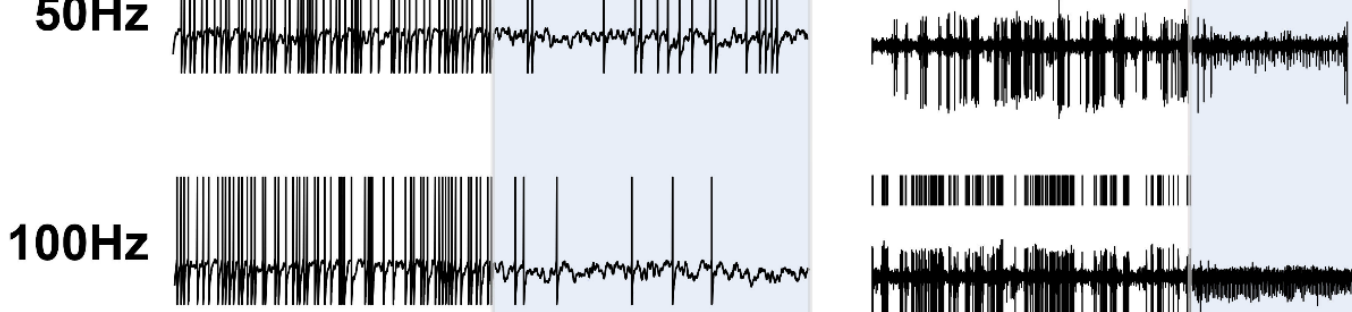

| | |||||||||| |||||| | ||||||||| | ||||| |||||||
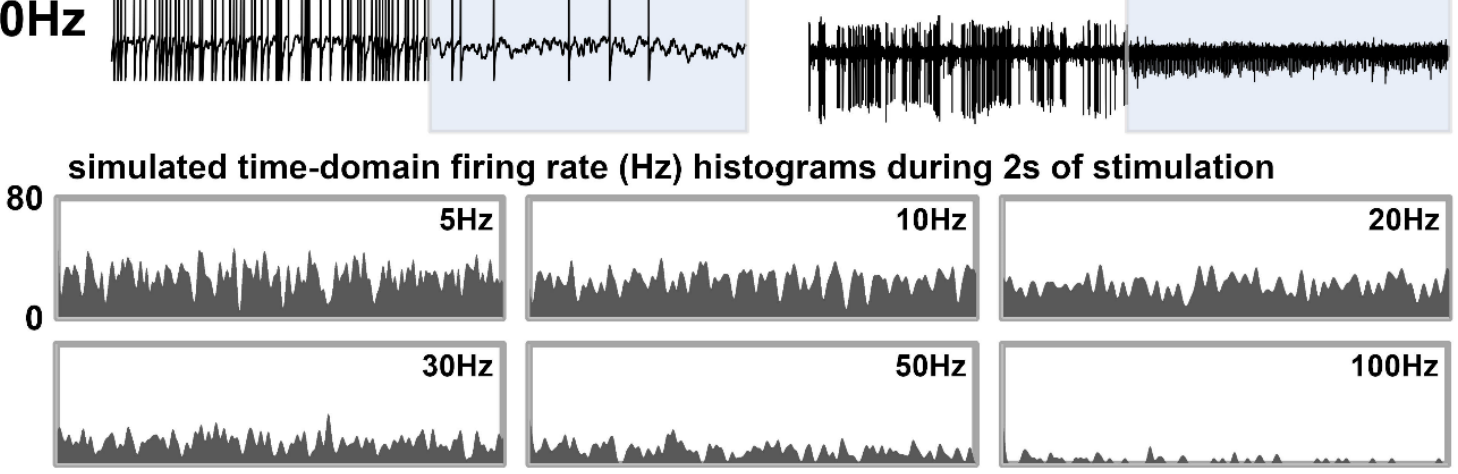

Fig. 6 - Computational time-domain membrane potential for STN. The left panels show the membrane potential of a model STN neuron immediately before (non-shaded) and during (shaded) DBS across a range of frequencies. The right panels are exemplary recordings from an in vivo human STN neuron (stimulation for $50 \mathrm{~Hz}$ was limited to $1 \mathrm{~s}$ ). The bottom-most panels are time-domain firing rate histograms generated by averaging across 10 model STN neurons. The neuronal firing rate compared to baseline decreases for DBS at $\geq 30 \mathrm{~Hz}$, corroborating experimental data. The modelled neuronal firing rates are substantially attenuated with $50 \mathrm{~Hz}$ and $100 \mathrm{~Hz}$ (as is the case experimentally) due to non-selective synaptic depression. 


\section{SNr - simulated and real neuronal activity}

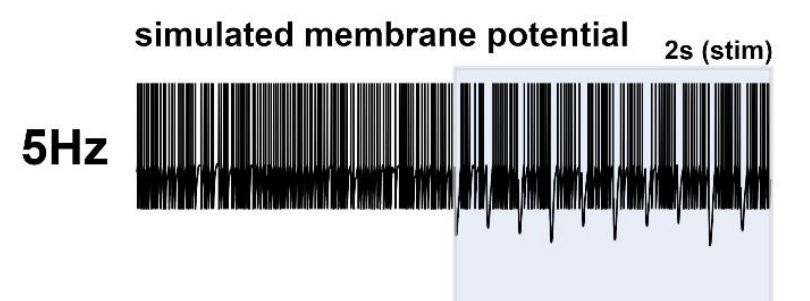

in vivo extracellular recording $2 \mathrm{~s}$ (stim)
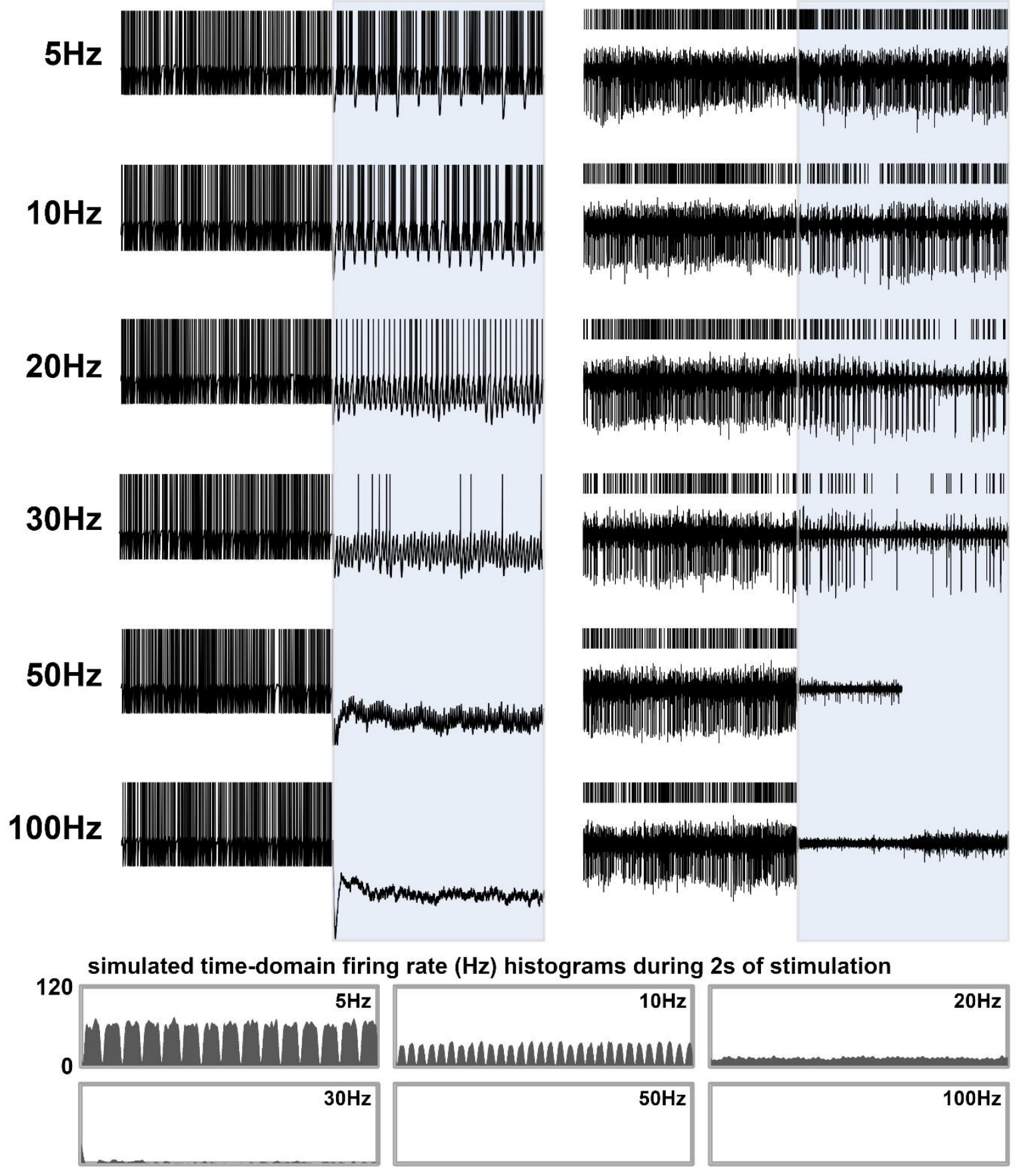

Fig. 7 - Computational time-domain membrane potential for SNr. The left panels show the membrane potential of a model SNr neuron immediately before (non-shaded) and during (shaded) DBS across a range of frequencies. The right panels are exemplary recordings from an in vivo human $\mathrm{SNr}$ neuron (stimulation for $50 \mathrm{~Hz}$ was limited to 1s). The bottom-most panels are time-domain firing rate histograms generated by averaging across 10 model $\mathrm{SNr}$ neurons. Due to the dominant inhibitory presynaptic currents, the neuronal firing rate decreases dramatically beginning at $20 \mathrm{~Hz}$, corroborating experimental data. The model neuron fails to generate action potentials for DBS $\geq 50 \mathrm{~Hz}$ (as is the case experimentally) due to non-selective synaptic depression 


\section{Methods}

\section{Experimental: Patients and neurons}

115 neurons from patients with Parkinson's disease $(n=45)$ or essential tremor $(n=14)$ were included in this study for the various analyses which are detailed below. All experiments conformed to the guidelines set by the Tri-Council Policy on Ethical Conduct for Research Involving Humans and were approved by the University Health Network Research Ethics Board. Moreover, each patient provided written informed consent prior to taking part in the studies.

\section{Experimental: Protocols}

Neurophysiological mapping procedures were performed during awake DBS surgeries (after overnight withdrawal from medications) using two closely spaced microelectrodes $(600 \mu \mathrm{m}$ apart, $0.1-0.4 \mathrm{M} \Omega$ impedances) (Levy et al., 2007). Techniques for identification of neurons of the Rt, STN, SNr (Hutchison et al., 1998), and Vim (Basha et al., 2014; Milosevic et al., 2018b) have been previously reported. While recording from a well-isolated single neuron (in one of the aforementioned structures) using one microelectrode, microstimulation was delivered at different frequencies using the adjacent microelectrode. Recordings were obtained using two Guideline System GS3000 amplifiers (Axon Instruments, Union City, CA) and signals were digitized at $\geq 12.5 \mathrm{kHz}$ with a CED 1401 data acquisition system (Cambridge Electronic Design, Cambridge, UK). Microstimulation was delivered using an isolated constant-current stimulator (Neuro-Amp1A, Axon Instruments, Union City, CA) with symmetric 0.3ms biphasic pulses (cathodal followed by anodal).

To generate stimulation frequency response functions, stimulation trains were delivered at $1 \mathrm{~Hz}(10$ pulses), $2 \mathrm{~Hz}$ (20 pulses), 3Hz (60 pulses), $5 \mathrm{~Hz}$ (50 pulses), 10Hz (50 pulses), $20 \mathrm{~Hz}$ (60 pulses), $30 \mathrm{~Hz}$ (60 pulses), $50 \mathrm{~Hz}$ (50 pulses), and $100 \mathrm{~Hz}$ (50 pulses) using $100 \mu \mathrm{A}$ and a $0.3 \mathrm{~ms}$ biphasic pulse width. This frequency response protocol was executed at $9 \mathrm{Vim}\left(\mathrm{n}_{\text {patients }}=5\right), 11 \mathrm{Rt}\left(\mathrm{n}_{\text {patients }}=11\right), 27 \mathrm{STN}\left(\mathrm{n}_{\text {patients }}=16\right)$, and $14 \mathrm{SNr}\left(\mathrm{n}_{\text {patients }}=9\right)$ recording sites. The average firing rates of all structures during stimulation at each frequency were plotted. The data for STN and SNr were previously collected (Milosevic et al., 2018a), whereas Vim and Rt data for this study were unique. Longer trains $(\geq 3 \mathrm{~s})$ of $100 \mathrm{~Hz}$ stimulation were also delivered to the aforementioned Vim, Rt, and SNr neurons. 100Hz long train data for STN (44 neurons, $\mathrm{n}_{\text {patients }}=20$ ) were previously collected (Milosevic et al., 2019), as were a subset of $100 \mathrm{~Hz}$ and $200 \mathrm{~Hz} \mathrm{Vim}$ (14 recording sites, $\mathrm{n}_{\text {patients }}=9$ ) data (Milosevic et al., 2018b). 


\section{Experimental: Offline analyses and statistics}

The narrow stimulus artifacts were removed offline ( $0.5 \mathrm{~ms}$ from stimulus onset), recordings were high pass filtered $(\geq 300 \mathrm{~Hz})$ to better isolate the single units, and template matching was done using a principal component analysis method in Spike2 (Cambridge Electronic Design, UK). For the frequency response protocol (in which $\leq 60$ stimulation pulses were delivered at each frequency), the average pre-stimulation baseline firing rates were measured, as were the firing rates during the various stimulation trains. Kolmogorov-Smirnov tests confirmed normality of data. One-way repeated measures ANOVA tests (stimulation frequency as a within-subject factor) were carried out, and in the event of a significant main effects, Bonferroni-corrected (nine comparisons) post hoc t-tests were used to compare firing rates during the various stimulation trains to the pre-stimulation baseline firing. ANOVA effect sizes ( $\eta^{2}$; proportion of variance associated with the main effects) and t-test effect sizes (Cohen's $\mathrm{d}_{\mathrm{z}}$; standardised difference between two means) were also determined. One neuron from the Vim group and one neuron from the Rt group were excluded from statistical analyses due to missing data points (i.e. incomplete stimulation protocol). To investigate the effects of individual stimulation pulses, an average peristimulus histogram (120ms total width, 20ms offset, $2 \mathrm{~ms}$ bins) was created for all neurons using the $5 \mathrm{~Hz}$ stimulation trains (50 pulses). The average firing rates during the $20 \mathrm{~ms}$ pre-stimulus periods were compared to the average firing rates during $20 \mathrm{~ms}$ and 40ms post-stimulus periods using Bonferroni-corrected (two comparisons) two-tailed paired t-tests, and effect sizes (Cohen's $d_{z}$ ) were calculated. Finally, to investigate possible time-varying responses throughout the duration of stimulation trains (rather than average responses to individual stimuli), time-series histograms (2-3s total width, no offset, $50 \mathrm{~ms}$ bins) were created for $5 \mathrm{~Hz}$, $10 \mathrm{~Hz}, 20 \mathrm{~Hz}, 30 \mathrm{~Hz}$, and long trains of $100 \mathrm{~Hz}$ (and $200 \mathrm{~Hz}$ long trains for Vim) stimulation trains (i.e. trains where $\geq 2 \mathrm{~s}$ of stimulation was delivered). The attenuations of excitation over time in Vim and Rt during stimulation trains of $\geq 20 \mathrm{~Hz}$ were modelled by double exponential functions. Similar histograms were also created for the shorter trains $(\leq 1 \mathrm{~s})$ of stimulation at $50 \mathrm{~Hz}$ and $100 \mathrm{~Hz}(0.5-1 \mathrm{~s}$ total width, no offset, $20 \mathrm{~ms}$ bins); these are available in Supplementary Fig. 1.

\section{Computational: Model framework}

To model the effect of DBS pulses on the afferents of the stimulated nuclei, we used a leaky integrate and fire (LIF) single neuron model, together with a TM model of short-term synaptic plasticity (Tsodyks et al., 1998). Each model neuron received a certain number of excitatory and inhibitory presynaptic inputs whose proportion was obtained using morphological data (ex. (Bottcher, 1975)). In addition to these 
inputs, the background synaptic activity (Destexhe et al., 2001) was modeled by an Ornstein-Uhlenbeck $(\mathrm{OU})$ process and added to the model neuron to reproduce the impact of synaptic noise that exists in vivo (Destexhe et al., 2001, 2003). In accordance with our first hypothesis, each DBS single pulse simultaneously activated all presynaptic inputs (Fig. 3A). This simultaneous activation was modeled by artificially generating precise spike times which correspond to the arrival of each DBS pulse in the presynaptic inputs. We utilized our modeling framework to recreate the neuronal firing in Vim, STN, and $\mathrm{SNr}$ in response to stimulation trains with frequencies of 1, 2, 5, 10, 20, 30, 50, 100Hz. Model generation for Rt neurons was omitted due to redundancy as the model parameters are identical to Vim except for the parameter which underlies the baseline firing rate (elaborated upon in the "Computational: Parameter settings" subsection below).

\section{Computational: Presynaptic inputs}

The vast majority of inputs to the Vim are glutamatergic projections from the dentate nucleus of the cerebellum (Asanuma et al., 1983; Ilinsky and Kultas-Ilinsky, 2002; Kultas-Ilinsky and Ilinsky, 1991; Kuramoto et al., 2011) and reciprocal connections from cerebral cortex (Kakei et al., 2001; Stepniewska et al., 1994), with less prominent inputs coming via inhibitory Rt projections (Ambardekar et al., 1999; Ilinsky et al., 1999; Kuramoto et al., 2011). The Rt is a thin sheet of neurons that forms a shell around the lateral and anterior borders of the dorsal, and to some extent ventral thalamus (Jones, 1975). It is primarily innervated by collateral branches of glutamatergic thalamocortical and corticothalamic projections (Crabtree, 1992a, 1992b; Gonzalo-Ruiz and Lieberman, 1995; Jones, 1975; Pinault, 2004), but also receives less prominent GABAergic innervation from the GPe and SNr (Asanuma, 1994; Hazrati and Parent, 1991; Pare' et al., 1990); like Vim, the majority of afferent inputs to Rt are glutamatergic. The vast majority ( $90 \%$ ) of inputs to the SNr are GABAergic, projecting from the striatum and globus pallidus externus (GPe) (Bolam et al., 2000; Parent and Hazrati, 1995), whereas the STN receives a more homogenous convergence of GABAergic and glutamatergic inputs from the GPe (Baufreton et al., 2009) and motor cortical areas (Nambu et al., 2002) respectively (Parent and Hazrati, 1995; Rinvik and Ottersen, 1993). While the mixed inputs are more homogenous in STN, electron-microscopy work suggests that GABAergic terminals nevertheless outnumber glutamatergic terminals (Kita and Kita, 2012). Estimates of the proportions of inhibitory and excitatory inputs are provided in Supplementary Table 1.

In the model, an ensemble of 500 neurons, each of which was modeled by an LIF model, produced inputs to the stimulated nuclei. Each neuron receiving a random input (modeled by OU process of time constant 
$5 \mathrm{~ms}$ ) fired at the rate of about $5 \mathrm{~Hz}$ (the total average firing rate across neurons is equal to $5 \pm 0.7 \mathrm{~Hz}$ ). Given an estimated proportion of excitation and inhibition, these neurons were labeled as excitatory and inhibitory neurons, and their spikes were fed to the stimulated nuclei through the TM model. The total synaptic current was obtained as a linear combination of presynaptic excitatory $\left(\mathrm{I}_{\mathrm{exc}}\right)$ and inhibitory currents $\left(\mathrm{I}_{\mathrm{inh}}\right)$ :

$I_{\text {syn }}(t)=w_{\text {exc }} I_{\text {exc }}(t)+w_{\text {inh }} I_{\text {inh }}(t)$

where $w_{\text {exc }}$ and $w_{i n h}$ denote the weights of excitatory and inhibitory currents, respectively. These weights, together with the mean and standard deviation of the background synaptic current, were tuned to reproduce the neuronal firing rates at the baseline (DBS-OFF) as well as in response to DBS with different frequencies (Supplementary Table 2).

\section{Computational: Synapse model}

We utilized the TM equations to model the function of short-term synaptic plasticity:

$\frac{d u}{d t}=-\frac{u}{\tau_{F}}+U\left(1-u^{-}\right) \delta\left(t-t_{s p}\right)$

$\frac{d r}{d t}=\frac{1-r}{\tau_{D}}+u^{+} r^{-} \delta\left(t-t_{s p}\right)$

$\frac{d I}{d t}=-\frac{I}{\tau_{s}}+A u^{+} r^{-} \delta\left(t-t_{s p}\right)$

where $u$ indicates utilization probability, i.e., the probability of releasing neurotransmitters in synaptic cleft due to calcium ion flux in the presynaptic terminal. Upon the arrival of each presynaptic spike, $t_{s p}, u$ increases by $U\left(1-u^{-}\right)$and then decays to zero by the facilitation time constant, $\tau_{f}$. As well, the vesicle depletion process - due to the release of neurotransmitters - was modeled by (2) where $r$ denotes the fraction of available resources after neurotransmitter depletion. In contrast to the increase of $u$ upon the arrival of each presynaptic spike, $r$ drops and then recovers by depression time constant $\tau_{D}$ to its steady state value of 1 . The competition between the depression $\left(\tau_{D}\right)$ and facilitation $\left(\tau_{f}\right)$ time constants determines the dynamics of the synapse. In the TM model, $U, \tau_{f}$, and $\tau_{D}$ are three parameters that determine the type and dynamics of the synapse. In (4), $I$ and $\tau_{s}$ indicate the presynaptic current and its time constant, respectively. The time constants of the excitatory and inhibitory inputs are $3 \mathrm{~ms}$ and $10 \mathrm{~ms}$, respectively. 


\section{Computational: Background synaptic activity}

Similar to (Destexhe et al., 2001), we used OU process of the time constant of $5 \mathrm{~ms}$ to represent the effect of synaptic noise. The OU process can be written as:

$\frac{d x}{d t}=-\frac{x(t)-\mu}{\tau}+a \sqrt{\frac{2}{\tau}} \xi(t)$

where $\xi$ is a random number drawn from a Gaussian distribution with 0 average and unit variance. $\tau$ is the time constant, $\mu$ and $\alpha$ indicate the mean and standard deviation of variable $x$, respectively.

\section{Computational: Neuron model}

The dynamics of membrane potential in an LIF model can be written as:

$\frac{d V(t)}{d t}=\frac{-\left(V(t)-E_{L}\right)+R I_{i n j}(t)}{\tau_{V}}$

where $E_{\mathrm{L}}=-70 \mathrm{mV}, R=1 \mathrm{M} \Omega$, and $\tau_{V}=10 \mathrm{~ms}$. $I_{i n j}$ indicates the total injected current to the model neuron (i.e., $I_{\text {syn }}$ plus background synaptic noise (5)). Spike occurs when $\mathrm{V} \geq \mathrm{Vth}$, where $V_{t h}=-40 \mathrm{mV}$ and the reset voltage is $-90 \mathrm{mV}$ with an absolute refractory period of $1 \mathrm{msec}$.

\section{Computational: Parameter settings}

The proportions of excitatory and inhibitory neurons (Supplementary Table 1), total synaptic current (Supplementary Table 2), parameters of excitatory (Supplementary Table 3) and inhibitory (Supplementary Table 4) synapses, and time constants of membrane dynamics and synaptic currents (Supplementary Table 5) are available in the Supplementary Material. As mentioned, modelling of Rt was omitted due to redundancy as all parameters are identical to Vim except for $\mathrm{w}_{\mathrm{exc}}$, winh (but with almost the same ratio) and parameters of background synaptic noise (Supplementary Table 2) which would mediate the difference in baseline firing between Rt and Vim. Parameters for Rt modelling are nevertheless included within the Supplementary Tables.

\section{Resource availability}

Anonymized experimental data is enclosed within Supplementary Data. The Codes for the computational model are available at https://github.com/nsbspl/DBS_Mechanism_Cellular. 


\section{Acknowledgements}

The authors thank Tameem Al-Ozzi for assistance in data collection and the patients for their participation.

\section{Author contributions}

1) Research project: A. Conception, B. Organization, C. Execution.

2) Data and statistical analysis: A. Design, B. Execution, C. Review and Critique.

3) Manuscript: A. Writing of the first draft, B. Review and Critique.

LM: 1A, 1B, 1C, 2A, 2B, 3A

SKK: 1B, 1C, 2C, 3B

MH: 1B, 1C, 2C, 3B

AML: 1B, 1C, 2C, 3B

MRP: 1B, 2C, 3B

WDH: 1A, 1B, 1C, 2C, 3B

ML: 1A, 1B, 1C, 2A, 2B, 3A

\section{Declaration of interests}

S.K.K., M.H., W.D.H. have received honoraria, travel funds, and/or grant support from Medtronic (not related to this work). A.M.L. has received honoraria, travel funds, and/or grant support from Medtronic, Boston Scientific, St. Jude-Abbott, and Insightec (not related to this work). M.R.P. is a shareholder in MyndTec Inc. A.M.L. is a co-founder of Functional Neuromodulation Ltd. L.M. and M.L. have no financial disclosures.

\section{Funding}

This work was supported in part by the Natural Sciences and Engineering Research Council: Discovery Grant RGPIN-2016-06358 (M.R.P.), Dean Connor and Maris Uffelmann Donation (M.R.P.), Walter \& Maria Schroeder Donation (M.R.P.), and the Dystonia Medical Research Foundation (W.D.H.). 


\section{Supplementary material information}

\section{Supplementary material}

Supplementary Table 1 - Proportions of excitatory and inhibitory presynaptic inputs

Supplementary Table 2 - Parameters for total current fed to the neuron

Supplementary Table 3 - Parameters of excitatory synapses

Supplementary Table 4 - Parameters of inhibitory synapses

Supplementary Table 5 - Time constants of membrane dynamics and synaptic currents

Supplementary Fig. 1 - Time-domain firing histograms for short trains of $50 \mathrm{~Hz}$ and $100 \mathrm{~Hz}$

Supplementary Fig. 2 - Induction of neuronal oscillation with low-frequency stimulation

\section{Supplementary data}

Experimental data files 


\section{References}

Albin, R.L., Young, A.B., and Penney, J.B. (1989). The functional anatomy of basal ganglia disorders. Trends in Neurosciences 12, $366-375$.

Alexander, G.E., and Crutcher, M.D. (1990). Functional architecture of basal ganglia circuits: neural substrates of parallel processing. Trends in Neurosciences 13, 266-271.

Alhourani, A., McDowell, M.M., Randazzo, M.J., Wozny, T.A., Kondylis, E.D., Lipski, W.J., Beck, S., Karp, J.F., Ghuman, A.S., and Richardson, R.M. (2015). Network effects of deep brain stimulation. Journal of Neurophysiology 114, $2105-2117$.

Ambardekar, A.V., Ilinsky, I.A., Froestl, W., Bowery, N.G., and Kultas-Ilinsky, K. (1999). Distribution and properties of GABAB antagonist [3H]CGP 62349 binding in the rhesus monkey thalamus and basal ganglia and the influence of lesions in the reticular thalamic nucleus. Neuroscience 93, 1339-1347.

Anderson, R.W., Farokhniaee, A., Gunalan, K., Howell, B., and McIntyre, C.C. (2018). Action potential initiation, propagation, and cortical invasion in the hyperdirect pathway during subthalamic deep brain stimulation. Brain Stimulation 11, 1140-1150.

Anderson, T., Hu, B., Pittman, Q., and Kiss, Z.H.T. (2004). Mechanisms of deep brain stimulation: an intracellular study in rat thalamus. The Journal of Physiology 559, 301-313.

Arnulf, I., Ferraye, M., Fraix, V., Benabid, A.L., Chabardès, S., Goetz, L., Pollak, P., and Debû, B. (2010). Sleep induced by stimulation in the human pedunculopontine nucleus area. Annals of Neurology 67, 546-549.

Asanuma, C. (1994). GABAergic and pallidal terminals in the thalamic reticular nucleus of squirrel monkeys. Exp Brain Res 101, $439-451$.

Asanuma, C., Thach, W.T., and Jones, E.G. (1983). Distribution of cerebellar terminations and their relation to other afferent terminations in the ventral lateral thalamic region of the monkey. Brain Research Reviews 5, 237-265.

Axelrad, J.E., Louis, E.D., Honig, L.S., Flores, I., Ross, G.W., Pahwa, R., Lyons, K.E., Faust, P.L., and Vonsattel, J.P.G. (2008). Reduced Purkinje Cell Number in Essential Tremor: A Postmortem Study. Arch Neurol 65, 101-107.

Barnikol, U.B., Popovych, O.V., Hauptmann, C., Sturm, V., Freund, H.-J., and Tass, P.A. (2008). Tremor entrainment by patterned low-frequency stimulation. Philosophical Transactions of the Royal Society A: Mathematical, Physical and Engineering Sciences $366,3545-3573$.

Basha, D., Dostrovsky, J.O., Lopez Rios, A.L., Hodaie, M., Lozano, A.M., and Hutchison, W.D. (2014). Beta oscillatory neurons in the motor thalamus of movement disorder and pain patients. Experimental Neurology 261, 782-790.

Basu, I., Robertson, M.M., Crocker, B., Peled, N., Farnes, K., Vallejo-Lopez, D.I., Deng, H., Thombs, M., Martinez-Rubio, C., Cheng, J.J., et al. (2019). Consistent linear and non-linear responses to invasive electrical brain stimulation across individuals and primate species with implanted electrodes. Brain Stimulation 12, 877-892.

Baufreton, J., Kirkham, E., Atherton, J.F., Menard, A., Magill, P.J., Bolam, J.P., and Bevan, M.D. (2009). Sparse but Selective and Potent Synaptic Transmission From the Globus Pallidus to the Subthalamic Nucleus. Journal of Neurophysiology 102, $532-545$.

Bazhenov, M., Timofeev, I., Steriade, M., and Sejnowski, T.J. (1999). Self-sustained rhythmic activity in the thalamic reticular nucleus mediated by depolarizing GABA A receptor potentials. Nature Neuroscience 2, 168-174.

Bekar, L., Libionka, W., Tian, G.-F., Xu, Q., Torres, A., Wang, X., Lovatt, D., Williams, E., Takano, T., Schnermann, J., et al. (2008). Adenosine is crucial for deep brain stimulation-mediated attenuation of tremor. Nature Medicine 14, 75-80.

Bergman, H., Wichmann, T., and DeLong, M.R. (1990). Reversal of experimental parkinsonism by lesions of the subthalamic nucleus. Science 249, 1436-1438.

Blumenfeld, H. (2005). Cellular and Network Mechanisms of Spike-Wave Seizures. Epilepsia 46, 21-33. 
Bolam, J.P., Hanley, J.J., Booth, P. a. C., and Bevan, M.D. (2000). Synaptic organisation of the basal ganglia. The Journal of Anatomy 196, 527-542.

Bottcher, J. (1975). Morphology of the basal ganglia in Parkinson's disease. Acta Neurol. Scand., Suppl. 62, 1-87.

Boulet, S., Lacombe, E., Carcenac, C., Feuerstein, C., Sgambato-Faure, V., Poupard, A., and Savasta, M. (2006). Subthalamic Stimulation-Induced Forelimb Dyskinesias Are Linked to an Increase in Glutamate Levels in the Substantia Nigra Pars Reticulata. J. Neurosci. 26, 10768-10776.

Brocker, D.T., Swan, B.D., So, R.Q., Turner, D.A., Gross, R.E., and Grill, W.M. (2017). Optimized temporal pattern of brain stimulation designed by computational evolution. Science Translational Medicine 9.

Brown, P. (2003). Oscillatory nature of human basal ganglia activity: Relationship to the pathophysiology of Parkinson's disease. Movement Disorders 18, 357-363.

Campos, A.C.P., Kikuchi, D.S., Paschoa, A.F.N., Kuroki, M.A., Fonoff, E.T., Hamani, C., Pagano, R.L., and Hernandes, M.S. (2020). Unraveling the Role of Astrocytes in Subthalamic Nucleus Deep Brain Stimulation in a Parkinson's Disease Rat Model. Cell Mol Neurobiol.

Chang, W.J., Chang, W.P., and Shyu, B.C. (2017). Suppression of cortical seizures by optic stimulation of the reticular thalamus in PV-mhChR2-YFP BAC transgenic mice. Molecular Brain 10, 42.

Chiken, S., and Nambu, A. (2014). Disrupting neuronal transmission: mechanism of DBS? Front Syst Neurosci 8.

Chudy, D., Deletis, V., Almahariq, F., Marčinković, P., Škrlin, J., and Paradžik, V. (2018). Deep brain stimulation for the early treatment of the minimally conscious state and vegetative state: experience in 14 patients. Journal of Neurosurgery 128, 1189-1198.

Cox, C.L., Huguenard, J.R., and Prince, D.A. (1997). Nucleus reticularis neurons mediate diverse inhibitory effects in thalamus. PNAS 94, 8854-8859.

Crabtree, J.W. (1992a). The Somatotopic Organization Within the Cat's Thalamic Reticular Nucleus. European Journal of Neuroscience 4, 1352-1361.

Crabtree, J.W. (1992b). The Somatotopic Organization Within the Rabbit's Thalamic Reticular Nucleus. European Journal of Neuroscience 4, 1343-1351.

Dallapiazza, R.F., Lee, D.J., Vloo, P.D., Fomenko, A., Hamani, C., Hodaie, M., Kalia, S.K., Fasano, A., and Lozano, A.M. (2019). Outcomes from stereotactic surgery for essential tremor. J Neurol Neurosurg Psychiatry 90, 474-482.

DeLong, M.R. (1990). Primate models of movement disorders of basal ganglia origin. Trends in Neurosciences 13, $281-285$.

Destexhe, A., Rudolph, M., Fellous, J.-M., and Sejnowski, T.J. (2001). Fluctuating synaptic conductances recreate in vivo-like activity in neocortical neurons. Neuroscience 107, 13-24.

Destexhe, A., Rudolph, M., and Paré, D. (2003). The high-conductance state of neocortical neurons in vivo. Nat Rev Neurosci 4, 739-751.

Dittman, J.S., and Regehr, W.G. (1998). Calcium Dependence and Recovery Kinetics of Presynaptic Depression at the Climbing Fiber to Purkinje Cell Synapse. J. Neurosci. 18, 6147-6162.

Dostrovsky, J.O., Levy, R., Wu, J.P., Hutchison, W.D., Tasker, R.R., and Lozano, A.M. (2000). Microstimulation-Induced Inhibition of Neuronal Firing in Human Globus Pallidus. Journal of Neurophysiology 84, 570-574.

Farokhniaee, A., and McIntyre, C.C. (2019). Theoretical principles of deep brain stimulation induced synaptic suppression. Brain Stimulation 12, 1402-1409. 
Fioravante, D., and Regehr, W.G. (2011). Short-term forms of presynaptic plasticity. Current Opinion in Neurobiology 21, 269274.

Fisher, R., Salanova, V., Witt, T., Worth, R., Henry, T., Gross, R., Oommen, K., Osorio, I., Nazzaro, J., Labar, D., et al. (2010). Electrical stimulation of the anterior nucleus of thalamus for treatment of refractory epilepsy. Epilepsia 51, 899-908.

Galati, S., Mazzone, P., Fedele, E., Pisani, A., Peppe, A., Pierantozzi, M., Brusa, L., Tropepi, D., Moschella, V., Raiteri, M., et al. (2006). Biochemical and electrophysiological changes of substantia nigra pars reticulata driven by subthalamic stimulation in patients with Parkinson's disease. European Journal of Neuroscience 23, 2923-2928.

Gentet, L.J., and Ulrich, D. (2003). Strong, reliable and precise synaptic connections between thalamic relay cells and neurones of the nucleus reticularis in juvenile rats. The Journal of Physiology 546, 801-811.

Gentet, L.J., and Ulrich, D. (2004). Electrophysiological characterization of synaptic connections between layer VI cortical cells and neurons of the nucleus reticularis thalami in juvenile rats. European Journal of Neuroscience 19, 625-633.

Gonzalo-Ruiz, A., and Lieberman, A.R. (1995). GABAergic projections from the thalamic reticular nucleus to the anteroventral and anterodorsal thalamic nuclei of the rat. Journal of Chemical Neuroanatomy 9, 165-174.

Hashimoto, T., Elder, C.M., Okun, M.S., Patrick, S.K., and Vitek, J.L. (2003). Stimulation of the Subthalamic Nucleus Changes the Firing Pattern of Pallidal Neurons. J. Neurosci. 23, 1916-1923.

Hazrati, L.-N., and Parent, A. (1991). Projection from the external pallidum to the reticular thalamic nucleus in the squirrel monkey. Brain Research 550, 142-146.

Helmich, R.C., Toni, I., Deuschl, G., and Bloem, B.R. (2013). The Pathophysiology of Essential Tremor and Parkinson's Tremor. Current Neurology and Neuroscience Reports 13 .

Hung, S.W., Hamani, C., Lozano, A.M., Poon, Y.-Y.W., Piboolnurak, P., Miyasaki, J.M., Lang, A.E., Dostrovsky, J.O., Hutchison, W.D., and Moro, E. (2007). Long-term outcome of bilateral pallidal deep brain stimulation for primary cervical dystonia. Neurology 68, 457.

Hutchison, W.D., Allan, R.J., Opitz, H., Levy, R., Dostrovsky, J.O., Lang, A.E., and Lozano, A.M. (1998). Neurophysiological identification of the subthalamic nucleus in surgery for Parkinson's disease. Annals of Neurology 44, $622-628$.

Ilinsky, I.A., and Kultas-Ilinsky, K. (2002). Motor thalamic circuits in primates with emphasis on the area targeted in treatment of movement disorders. Movement Disorders 17, S9-S14.

Ilinsky, I.A., Ambardekar, A.V., and Kultas-Ilinsky, K. (1999). Organization of projections from the anterior pole of the nucleus reticularis thalami (NRT) to subdivisions of the motor thalamus: Light and electron microscopic studies in the Rhesus monkey. Journal of Comparative Neurology 409, 369-384.

Jakobs, M., Fomenko, A., Lozano, A.M., and Kiening, K.L. (2019). Cellular, molecular, and clinical mechanisms of action of deep brain stimulation - a systematic review on established indications and outlook on future developments. EMBO Molecular Medicine 11 .

Jones, E.G. (1975). Some aspects of the organization of the thalamic reticular complex. Journal of Comparative Neurology 162, $285-308$.

Kakei, S., Na, J., and Shinoda, Y. (2001). Thalamic terminal morphology and distribution of single corticothalamic axons originating from layers 5 and 6 of the cat motor cortex. Journal of Comparative Neurology 437, 170-185.

Kane, A., Hutchison, W.D., Hodaie, M., Lozano, A.M., and Dostrovsky, J.O. (2009). Enhanced synchronization of thalamic theta band local field potentials in patients with essential tremor. Experimental Neurology 217, 171-176.

Kim, U., Sanchez-Vives, M.V., and McCormick, D.A. (1997). Functional Dynamics of GABAergic Inhibition in the Thalamus. Science 278, 130-134. 
Kita, T., and Kita, H. (2012). The Subthalamic Nucleus Is One of Multiple Innervation Sites for Long-Range Corticofugal Axons: A Single-Axon Tracing Study in the Rat. J. Neurosci. 32, 5990-5999.

Krauss, G.L., and Koubeissi, M.Z. (2007). Cerebellar and thalamic stimulation treatment for epilepsy. In Operative Neuromodulation: Volume 2: Neural Networks Surgery, D.E. Sakas, and B.A. Simpson, eds. (Vienna: Springer), pp. $347-356$.

Kühn, A.A., Kempf, F., Brücke, C., Doyle, L.G., Martinez-Torres, I., Pogosyan, A., Trottenberg, T., Kupsch, A., Schneider, G.-H., Hariz, M.I., et al. (2008). High-Frequency Stimulation of the Subthalamic Nucleus Suppresses Oscillatory $\beta$ Activity in Patients with Parkinson's Disease in Parallel with Improvement in Motor Performance. J. Neurosci. 28, 6165-6173.

Kultas-Ilinsky, K., and Ilinsky, I.A. (1991). Fine structure of the ventral lateral nucleus (VL) of the Macaca mulatta thalamus: Cell types and synaptology. Journal of Comparative Neurology 314, 319-349.

Kultas-Ilinsky, K., Yi, H., and Ilinsky, I.A. (1995). Nucleus reticularis thalami input to the anterior thalamic nuclei in the monkey: a light and electron microscopic study. Neuroscience Letters 186, 25-28.

Kuramoto, E., Fujiyama, F., Nakamura, K.C., Tanaka, Y., Hioki, H., and Kaneko, T. (2011). Complementary distribution of glutamatergic cerebellar and GABAergic basal ganglia afferents to the rat motor thalamic nuclei. European Journal of Neuroscience $33,95-109$.

Landisman, C.E., Long, M.A., Beierlein, M., Deans, M.R., Paul, D.L., and Connors, B.W. (2002). Electrical Synapses in the Thalamic Reticular Nucleus. J. Neurosci. 22, 1002-1009.

Lavian, H., Loewenstern, Y., Madar, R., Almog, M., Bar-Gad, I., Okun, E., and Korngreen, A. (2018). Dopamine receptors in the rat entopeduncular nucleus. Brain Struct Funct 223, 2673-2684.

Lavoie, B., Smith, Y., and Parent, A. (1989). Dopaminergic innervation of the basal ganglia in the squirrel monkey as revealed by tyrosine hydroxylase immunohistochemistry. Journal of Comparative Neurology 289, 36-52.

Lenz, F.A., Tasker, R.R., Kwan, H.C., Schnider, S., Kwong, R., Murayama, Y., Dostrovsky, J.O., and Murphy, J.T. (1988). Single unit analysis of the human ventral thalamic nuclear group: correlation of thalamic "tremor cells" with the 3-6 Hz component of parkinsonian tremor. J. Neurosci. 8, 754-764.

Levy, R., Lang, A.E., Dostrovsky, J.O., Pahapill, P., Romas, J., Saint-Cyr, J., Hutchison, W.D., and Lozano, A.M. (2001). Lidocaine and muscimol microinjections in subthalamic nucleus reverse parkinsonian symptoms. Brain 124, $2105-2118$.

Levy, R., Lozano, A.M., Hutchison, W.D., and Dostrovsky, J.O. (2007). Dual Microelectrode Technique for Deep Brain Stereotactic Surgery in Humans. Oper Neurosurg (Hagerstown) 60, ONS-277-ONS-284.

Lewis, L.D., Voigts, J., Flores, F.J., Schmitt, L.I., Wilson, M.A., Halassa, M.M., and Brown, E.N. (2015). Thalamic reticular nucleus induces fast and local modulation of arousal state. ELife 4, e08760.

Lim, A.S., Moro, E., Lozano, A.M., Hamani, C., Dostrovsky, J.O., Hutchison, W.D., Lang, A.E., Wennberg, R.A., and Murray, B.J . (2009). Selective enhancement of rapid eye movement sleep by deep brain stimulation of the human pons. Annals of Neurology 66, $110-114$.

Limousin, P., Krack, P., Pollak, P., Benazzouz, A., Ardouin, C., Hoffmann, D., and Benabid, A.-L. (1998). Electrical Stimulation of the Subthalamic Nucleus in Advanced Parkinson's Disease. New England Journal of Medicine 339, $1105-1111$.

Liu, L.D., Prescott, I.A., Dostrovsky, J.O., Hodaie, M., Lozano, A.M., and Hutchison, W.D. (2012). Frequency-dependent effects of electrical stimulation in the globus pallidus of dystonia patients. Journal of Neurophysiology 108, 5-17.

Louis, E.D., Faust, P.L., Vonsattel, J.-P.G., Honig, L.S., Rajput, A., Robinson, C.A., Rajput, A., Pahwa, R., Lyons, K.E., Ross, G.W., et al. (2007). Neuropathological changes in essential tremor: 33 cases compared with 21 controls. Brain 130, $3297-3307$.

Louis, E.D., Faust, P.L., Ma, K.J., Yu, M., Cortes, E., and Vonsattel, J.-P.G. (2011). Torpedoes in the Cerebellar Vermis in Essential Tremor Cases vs. Controls. Cerebellum 10, 812-819. 
Louis, E.D., Kerridge, C.A., Chatterjee, D., Martuscello, R.T., Diaz, D.T., Koeppen, A.H., Kuo, S.-H., Vonsattel, J.-P.G., Sims, P.A., and Faust, P.L. (2019). Contextualizing the pathology in the essential tremor cerebellar cortex: a patholog-omics approach. Acta Neuropathol 138, 859-876.

Lymer, J., Prescott, I.A., and Levy, R. (2019). Microstimulation-induced inhibition of thalamic reticular nucleus in non-human primates. Exp Brain Res 237, 1511-1520.

Maltête, D., Jodoin, N., Karachi, C., Houeto, J.L., Navarro, S., Cornu, P., Agid, Y., and Welter, M.L. (2007). Subthalamic Stimulation and Neuronal Activity in the Substantia Nigra in Parkinson's Disease. Journal of Neurophysiology $97,4017-4022$.

McAlonan, K., Cavanaugh, J., and Wurtz, R.H. (2008). Guarding the gateway to cortex with attention in visual thalamus. Nature $456,391-394$.

McIntyre, C.C., and Hahn, P.J. (2010). Network perspectives on the mechanisms of deep brain stimulation. Neurobiology of Disease 38, 329-337.

McIntyre, C.C., Grill, W.M., Sherman, D.L., and Thakor, N.V. (2004). Cellular Effects of Deep Brain Stimulation: Model-Based Analysis of Activation and Inhibition. Journal of Neurophysiology 91, 1457-1469.

Menchón, J.M., Real, E., Alonso, P., Aparicio, M.A., Segalas, C., Plans, G., Luyten, L., Brunfaut, E., Matthijs, L., Raymakers, S., et al. (2019). A prospective international multi-center study on safety and efficacy of deep brain stimulation for resistant obsessivecompulsive disorder. Molecular Psychiatry 1-14.

Milosevic, L., Kalia, S.K., Hodaie, M., Lozano, A.M., Fasano, A., Popovic, M.R., and Hutchison, W.D. (2018a). Neuronal inhibition and synaptic plasticity of basal ganglia neurons in Parkinson's disease. Brain 141, 177-190.

Milosevic, L., Kalia, S.K., Hodaie, M., Lozano, A.M., Popovic, M.R., and Hutchison, W.D. (2018b). Physiological mechanisms of thalamic ventral intermediate nucleus stimulation for tremor suppression. Brain 141, 2142-2155.

Milosevic, L., Kalia, S.K., Hodaie, M., Lozano, A., Popovic, M.R., and Hutchison, W. (2019). Subthalamic suppression defines therapeutic threshold of deep brain stimulation in Parkinson's disease. J Neurol Neurosurg Psychiatry 90, 1105-1108.

Moreau, C., Defebvre, L., Devos, D., Marchetti, F., Destée, A., Stefani, A., and Peppe, A. (2009). STN versus PPN-DBS for alleviating freezing of gait: Toward a frequency modulation approach? Movement Disorders 24, 2164-2166.

Nambu, A., Tokuno, H., and Takada, M. (2002). Functional significance of the cortico-subthalamo-pallidal 'hyperdirect' pathway. Neuroscience Research 43, 111-117.

Nanobashvili, Z., Chachua, T., Nanobashvili, A., Bilanishvili, I., Lindvall, O., and Kokaia, Z. (2003). Suppression of limbic motor seizures by electrical stimulation in thalamic reticular nucleus. Experimental Neurology 181, 224-230.

Nanobashvili, Z.I., Surmava, A.G., Bilanishvili, I.G., Barbaqadze, M.G., Mariamidze, M.D., and Khizanishvili, N.A. (2012). Significance of the Thalamic Reticular Nucleus GABAergic Neurons in Normal and Pathological Activity of the Brain. p.

Pantoja-Jiménez, C.R., Magdaleno-Madrigal, V.M., Almazán-Alvarado, S., and Fernández-Mas, R. (2014). Anti-epileptogenic Effect of High-frequency Stimulation in the Thalamic Reticular Nucleus on PTZ-induced Seizures. Brain Stimulation 7, $587-594$.

Pare', D., Hazrati, L.-N., Parent, A., and Steriade, M. (1990). Substantia nigra pars reticulata projects to the reticular thalamic nucleus of the cat: a morphological and electrophysiological study. Brain Research 535, 139-146.

Parent, A., and Hazrati, L.-N. (1995). Functional anatomy of the basal ganglia. II. The place of subthalamic nucleus and external pallidium in basal ganglia circuitry. Brain Research Reviews 20, 128-154.

Paris-Robidas, S., Brochu, E., Sintes, M., Emond, V., Bousquet, M., Vandal, M., Pilote, M., Tremblay, C., Di Paolo, T., Rajput, A.H., et al. (2012). Defective dentate nucleus GABA receptors in essential tremor. Brain 135, 105-116.

Pinault, D. (2004). The thalamic reticular nucleus: structure, function and concept. Brain Research Reviews 46, 1-31. 
Ran, I., Quastel, D.M.J., Mathers, D.A., and Puil, E. (2009). Fluctuation Analysis of Tetanic Rundown (Short-Term Depression) at a Corticothalamic Synapse. Biophysical Journal 96, 2505-2531.

Rinvik, E., and Ottersen, O.P. (1993). Terminals of subthalamonigral fibres are enriched with glutamate-like immunoreactivity: An electron microscopic, immunogold analysis in the cat. Journal of Chemical Neuroanatomy 6, 19-30.

Rizzoli, S.O., and Betz, W.J. (2005). Synaptic vesicle pools. Nature Reviews Neuroscience 6, 57-69.

Rosenbaum, R., Zimnik, A., Zheng, F., Turner, R.S., Alzheimer, C., Doiron, B., and Rubin, J.E. (2014). Axonal and synaptic failure suppress the transfer of firing rate oscillations, synchrony and information during high frequency deep brain stimulation. Neurobiology of Disease 62, 86-99.

Rosenmund, C., and Stevens, C.F. (1996). Definition of the Readily Releasable Pool of Vesicles at Hippocampal Synapses. Neuron 16, 1197-1207.

Salatino, J.W., Ludwig, K.A., Kozai, T.D.Y., and Purcell, E.K. (2017). Glial responses to implanted electrodes in the brain. Nature Biomedical Engineering 1, 862-877.

Schlag, J., and Waszak, M. (1971). Electrophysiological properties of units of the thalamic reticular complex. Experimental Neurology 32, 79-97.

Shill, H.A., Adler, C.H., Sabbagh, M.N., Connor, D.J., Caviness, J.N., Hentz, J.G., and Beach, T.G. (2008). Pathologic findin gs in prospectively ascertained essential tremor subjects. Neurology 70, 1452.

Steiner, L.A., Tomás, F.J.B., Planert, H., Alle, H., Vida, I., and Geiger, J.R.P. (2019). Connectivity and Dynamics Underlying Synaptic Control of the Subthalamic Nucleus. J. Neurosci. 39, 2470-2481.

Stepniewska, I., Preuss, T.M., and Kaas, J.H. (1994). Thalamic connections of the primary motor cortex (M1) of owl monkeys. Journal of Comparative Neurology 349, 558-582.

Steriade, M., Parent, A., and Hada, J. (1984). Thalamic projections of nucleus reticularis thalami of cat: A study using retrograde transport of horseradish peroxidase and fluorescent tracers. Journal of Comparative Neurology 229, 531-547.

Surmeier, D.J., Ding, J., Day, M., Wang, Z., and Shen, W. (2007). D1 and D2 dopamine-receptor modulation of striatal glutamatergic signaling in striatal medium spiny neurons. Trends in Neurosciences 30, 228-235.

Swan, B.D., Brocker, D.T., Hilliard, J.D., Tatter, S.B., Gross, R.E., Turner, D.A., and Grill, W.M. (2016). Short pauses in thalamic deep brain stimulation promote tremor and neuronal bursting. Clinical Neurophysiology 127, 1551-1559.

Tai, C.-H., Boraud, T., Bezard, E., Bioulac, B., Gross, C., and Benazzouz, A. (2003). Electrophysiological and metabolic evidence that high-frequency stimulation of the subthalamic nucleus bridles neuronal activity in the subthalamic nucleus and the substantia nigra reticulata. The FASEB Journal 17, 1820-1830.

Tawfik, V.L., Chang, S.-Y., Hitti, F.L., Roberts, D.W., Leiter, J.C., Jovanovic, S., and Lee, K.H. (2010). Deep Brain Stimulation Results in Local Glutamate and Adenosine Release: Investigation Into the Role of Astrocytes. Neurosurgery 67, 367-375.

Tsodyks, M.V., and Markram, H. (1997). The neural code between neocortical pyramidal neurons depends on neurotransmitter release probability. PNAS 94, 719-723.

Tsodyks, M., Pawelzik, K., and Markram, H. (1998). Neural Networks with Dynamic Synapses. Neural Computation 10, 821-835.

Ulrich, D., and Huguenard, J.R. (1995). Purinergic inhibition of GABA and glutamate release in the thalamus: Implications for thalamic network activity. Neuron 15,909-918.

Wang, Z., and Wang, Q. (2017). Eliminating Absence Seizures through the Deep Brain Stimulation to Thalamus Reticular Nucleus. Front. Comput. Neurosci. 11. 
Wichmann, T., Bergman, H., and DeLong, M.R. (1994). The primate subthalamic nucleus. III. Changes in motor behavior and neuronal activity in the internal pallidum induced by subthalamic inactivation in the MPTP model of parkinsonism. Journal of Neurophysiology 72, 521-530.

Yamamoto, T., and Katayama, Y. (2005). Deep brain stimulation therapy for the vegetative state. Neuropsychological Rehabilitation 15, 406-413.

Youngerman, B.E., Chan, A.K., Mikell, C.B., McKhann, G.M., and Sheth, S.A. (2016). A decade of emerging indications: deep brain stimulation in the United States. Journal of Neurosurgery 125, 461-471.

Yu, M., Ma, K., Faust, P.L., Honig, L.S., Cortés, E., Vonsattel, J.-P.G., and Louis, E.D. (2012). Increased number of Purkinje cell dendritic swellings in essential tremor. European Journal of Neurology 19, 625-630.

Zheng, F., Lammert, K., Nixdorf-Bergweiler, B.E., Steigerwald, F., Volkmann, J., and Alzheimer, C. (2011). Axonal failure during high frequency stimulation of rat subthalamic nucleus. The Journal of Physiology 589, 2781-2793.

Zucker, R.S., and Regehr, W.G. (2002). Short-Term Synaptic Plasticity. Annual Review of Physiology 64, 355-405. 